\title{
Concentration Inequalities and Martingale Inequalities: A Survey
}

Fan Chung and Linyuan Lu

Abstract. We examine a number of generalized and extended versions of concentration inequalities and martingale inequalities. These inequalities are effective for analyzing processes with quite general conditions as illustrated in an example for an infinite Polya process and web graphs.

\section{Introduction}

One of the main tools in probabilistic analysis is the concentration inequalities. Basically, the concentration inequalities are meant to give a sharp prediction of the actual value of a random variable by bounding the error term (from the expected value) with an associated probability. The classical concentration inequalities such as those for the binomial distribution have the best possible error estimates with exponentially small probabilistic bounds. Such concentration inequalities usually require certain independence assumptions (i.e., the random variable can be decomposed as a sum of independent random variables).

When the independence assumptions do not hold, it is still desirable to have similar, albeit slightly weaker, inequalities at our disposal. One approach is the martingale method. If the random variable and the associated probability space can be organized into a chain of events with modified probability spaces 
and if the incremental changes of the value of the event is "small," then the martingale inequalities provide very good error estimates. The reader is referred to numerous textbooks [Alon and Spencer 92, Janson et al. 00, McDiarmid 98] on this subject.

In the past few years, there has been a great deal of research in analyzing general random graph models for realistic massive graphs that have uneven degree distribution, such as the power law [Abello et al. 98, Aiello et al. 00, Aiello et al. 02, Albert and Barabási 02, Barabási and Albert 99]. The usual concentration inequalities and martingale inequalities have often been found to be inadequate and in many cases not feasible. The reasons are multifold: due to uneven degree distribution, the error bound of those very large degrees offset the delicate analysis in the sparse part of the graph. For the setup of the martingales, a uniform upper bound for the incremental changes are often too poor to be of any use. Furthermore, the graph is dynamically evolving, and therefore the probability space is changing at each tick of the time.

In spite of these difficulties, it is highly desirable to extend the classical concentration inequalities and martingale inequalities so that rigorous analysis for random graphs with general degree distributions can be carried out. Indeed, in the course of studying general random graphs, a number of variations and generalizations of concentration inequalities and martingale inequalities have been scattered around. It is the goal of this survey to put together these extensions and generalizations to present a more complete picture. We will examine and compare these inequalities, and complete proofs will be given. Needless to say, this survey is far from complete since all the work is quite recent and the selection is heavily influenced by our personal learning experience on this topic. Indeed, many of these inequalities have been included in our previous papers [Chung and Lu 02b, Chung and Lu 02a, Chung et al. 03b, Chung and Lu 04].

In addition to numerous variations of the inequalities, we also include an example of an application on a generalization of Polya's urn problem. Due to the fundamental nature of these concentration inequalities and martingale inequalities, they may be useful for many other problems as well.

This paper is organized as follows:

1. Introduction: overview, recent developments and summary.

2. Binomial distribution and its asymptotic behavior: the normalized binomial distribution and Poisson distribution.

3. General Chernoff inequalities: sums of independent random variables in five different concentration inequalities. 
4. More concentration inequalities: five more variations of the concentration inequalities.

5. Martingales and Azuma's inequality: basics for martingales and proofs for Azuma's inequality.

6. General martingale inequalities: four general versions of martingale inequalities with proofs.

7. Supermartingales and submartingales: modifying the definitions for martingale and still preserving the effectiveness of the martingale inequalities.

8. The decision tree and relaxed concentration inequalities: instead of the worst case incremental bound (the Lipschitz condition), only certain "local" conditions are required.

9. A generalized Polya's urn problem: an application for an infinite Polya process by using these general concentration and martingale inequalities. For web graphs generated by the preferential attachment scheme, the concentration for the power law degree distribution can be derived in a similar way.

\section{The Binomial Distribution and Its Asymptotic Behavior}

Bernoulli trials, named after James Bernoulli, can be thought of as a sequence of coin flips. For some fixed value $p$, where $0 \leq p \leq 1$, the outcome of the coin tossing process has probability $p$ of getting a "head." Let $S_{n}$ denote the number of heads after $n$ tosses. We can write $S_{n}$ as a sum of independent random variables $X_{i}$ as follows:

$$
S_{n}=X_{1}+X_{2}+\cdots+X_{n}
$$

where, for each $i$, the random variable $X$ satisfies

$$
\begin{aligned}
& \operatorname{Pr}\left(X_{i}=1\right)=p \\
& \operatorname{Pr}\left(X_{i}=0\right)=1-p .
\end{aligned}
$$

A classical question is to determine the distribution of $S_{n}$. It is not too difficult to see that $S_{n}$ has the binomial distribution $B(n, p)$ :

$$
\operatorname{Pr}\left(S_{n}=k\right)=\left(\begin{array}{l}
n \\
k
\end{array}\right) p^{k}(1-p)^{n-k}, \quad \text { for } k=0,1,2, \ldots, n .
$$




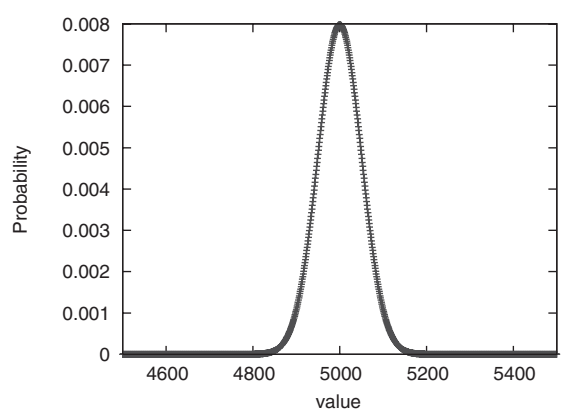

Figure I. The binomial distribution $B(10000,0.5)$.

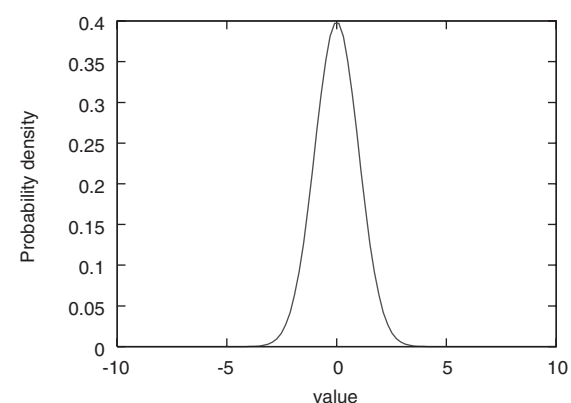

Figure 2. The standard normal distribution $N(0,1)$.

The expectation and variance of $B(n, p)$ are

$$
\mathrm{E}\left(S_{n}\right)=n p \quad \text { and } \quad \operatorname{Var}\left(S_{n}\right)=n p(1-p),
$$

respectively.

To better understand the asymptotic behavior of the binomial distribution, we compare it with the normal distribution $N(\alpha, \sigma)$, whose density function is given by

$$
f(x)=\frac{1}{\sqrt{2 \pi} \sigma} e^{-\frac{(x-\alpha)^{2}}{2 \sigma^{2}}}, \quad-\infty<x<\infty,
$$

where $\alpha$ denotes the expectation and $\sigma^{2}$ is the variance.

The case $N(0,1)$ is called the standard normal distribution whose density function is given by

$$
f(x)=\frac{1}{\sqrt{2 \pi}} e^{-x^{2} / 2}, \quad-\infty<x<\infty .
$$

When $p$ is a constant, the limit of the binomial distribution, after scaling, is the standard normal distribution and can be viewed as a special case of the Central-Limit Theorem, sometimes called the DeMoivre-Laplace limit Theorem [Feller 71].

Theorem 2.I. The binomial distribution $B(n, p)$ for $S_{n}$, as defined in (2.1), satisfies, for two constants $a$ and $b$,

$$
\lim _{n \rightarrow \infty} \operatorname{Pr}\left(a \sigma<S_{n}-n p<b \sigma\right)=\int_{a}^{b} \frac{1}{\sqrt{2 \pi}} e^{-x^{2} / 2} d x,
$$

where $\sigma=\sqrt{n p(1-p)}$ provided that $n p(1-p) \rightarrow \infty$ as $n \rightarrow \infty$. 


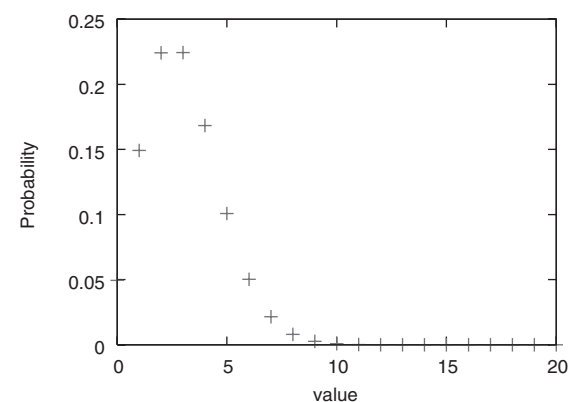

Figure 3. The binomial distribution $B(1000,0.003)$.

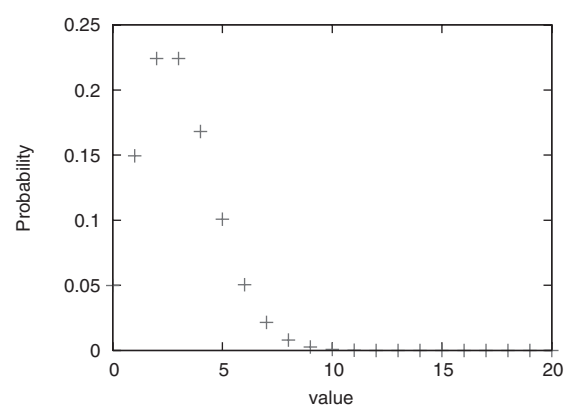

Figure 4. The Poisson distribution $P(3)$.

When $n p$ is upper bounded (by a constant), Theorem 2.1 is no longer true. For example, for $p=\frac{\lambda}{n}$, the limit distribution of $B(n, p)$ is the so-called Poisson distribution $P(\lambda)$ :

$$
\operatorname{Pr}(X=k)=\frac{\lambda^{k}}{k !} e^{-\lambda}, \quad \text { for } k=0,1,2, \cdots .
$$

The expectation and variance of the Poisson distribution $P(\lambda)$ are given by

$$
\mathrm{E}(X)=\lambda \quad \text { and } \quad \operatorname{Var}(X)=\lambda .
$$

Theorem 2.2. For $p=\frac{\lambda}{n}$, where $\lambda$ is a constant, the limit distribution of binomial distribution $B(n, p)$ is the Poisson distribution $P(\lambda)$.

Proof. We consider

$$
\begin{aligned}
\lim _{n \rightarrow \infty} \operatorname{Pr}\left(S_{n}=k\right) & =\lim _{n \rightarrow \infty}\left(\begin{array}{l}
n \\
k
\end{array}\right) p^{k}(1-p)^{n-k} \\
& =\lim _{n \rightarrow \infty} \frac{\lambda^{k} \prod_{i=0}^{k-1}\left(1-\frac{i}{n}\right)}{k !} e^{-p(n-k)} \\
& =\frac{\lambda^{k}}{k !} e^{-\lambda} .
\end{aligned}
$$

As $p$ decreases from $\Theta(1)$ to $\Theta\left(\frac{1}{n}\right)$, the asymptotic behavior of the binomial distribution $B(n, p)$ changes from the normal distribution to the Poisson distribution. (Some examples are illustrated in Figures 5 and 6.) Theorem 2.1 states that the asymptotic behavior of $B(n, p)$ within the interval $(n p-C \sigma, n p+C \sigma)$ (for any constant $C$ ) is close to the normal distribution. In some applications, we might need asymptotic estimates beyond this interval. 


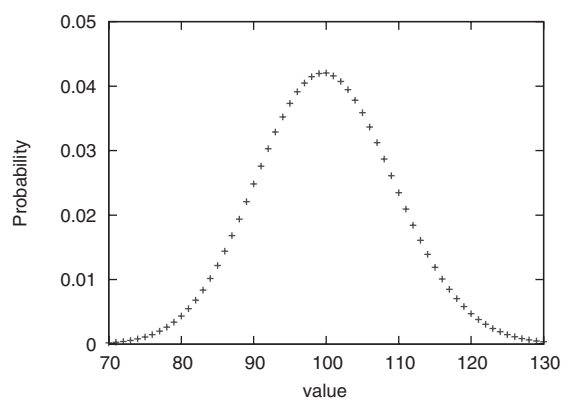

Figure 5. The binomial distribution $B(1000,0.1)$.

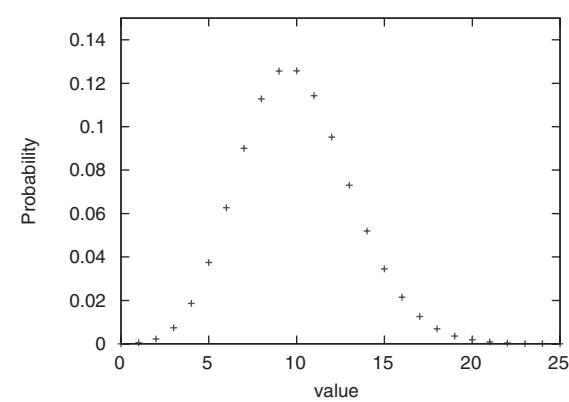

Figure 6. The binomial distribution $B(1000,0.01)$.

\section{General Chernoff Inequalities}

If the random variable under consideration can be expressed as a sum of independent variables, it is possible to derive good estimates. The binomial distribution is one such example where $S_{n}=\sum_{i=1}^{n} X_{i}$ and the $X_{i}$ are independent and identical. In this section, we consider sums of independent variables that are not necessarily identical. To control the probability of how close a sum of random variables is to the expected value, various concentration inequalities are in play. A typical version of the Chernoff inequalities, attributed to Herman Chernoff, can be stated as follows:

Theorem 3.I. [Chernoff 81] Let $X_{1}, \ldots, X_{n}$ be independent random variables with $\mathrm{E}\left(X_{i}\right)=0$ and $\left|X_{i}\right| \leq 1$ for all $i$. Let $X=\sum_{i=1}^{n} X_{i}$, and let $\sigma^{2}$ be the variance of $X_{i}$. Then,

$$
\operatorname{Pr}(|X| \geq k \sigma) \leq 2 e^{-k^{2} / 4 n},
$$

for any $0 \leq k \leq 2 \sigma$.

If the random variables $X_{i}$ under consideration assume nonnegative values, the following version of Chernoff inequalities is often useful.

Theorem 3.2. [Chernoff 81] Let $X_{1}, \ldots, X_{n}$ be independent random variables with

$$
\operatorname{Pr}\left(X_{i}=1\right)=p_{i}, \quad \operatorname{Pr}\left(X_{i}=0\right)=1-p_{i} .
$$

We consider the sum $X=\sum_{i=1}^{n} X_{i}$, with expectation $\mathrm{E}(X)=\sum_{i=1}^{n} p_{i}$. Then, we have

$$
\begin{array}{ll}
\text { (Lower tail) } & \operatorname{Pr}(X \leq \mathrm{E}(X)-\lambda) \leq e^{-\lambda^{2} / 2 \mathrm{E}(X)}, \\
\text { (Upper tail) } & \operatorname{Pr}(X \geq \mathrm{E}(X)+\lambda) \leq e^{-\frac{\lambda^{2}}{2(\mathrm{E}(X)+\lambda / 3)}} .
\end{array}
$$


Upper tails

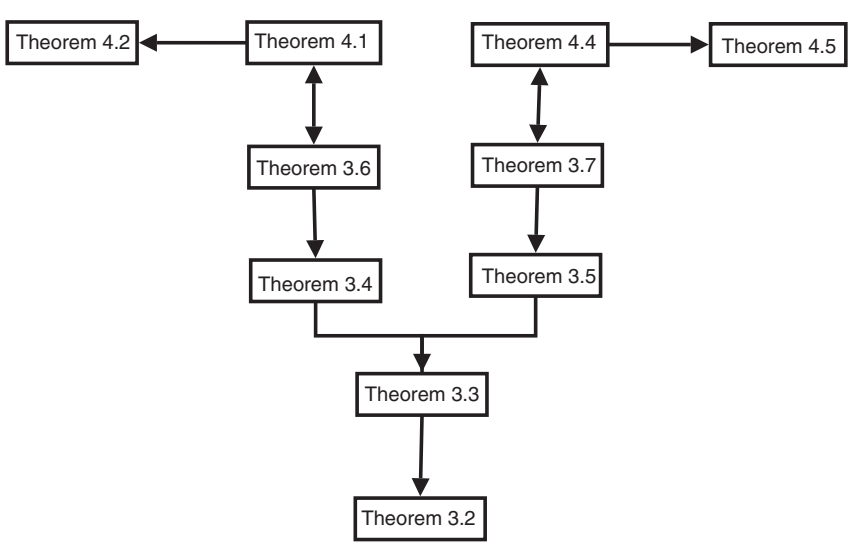

Figure 7. The flowchart for theorems on the sum of independent variables.

We remark that the term $\lambda / 3$ appearing in the exponent of the bound for the upper tail is significant. This covers the case when the limit distribution is Poisson as well as normal.

There are many variations of the Chernoff inequalities. Due to the fundamental nature of these inequalities, we will state several versions and then prove the strongest version from which all the other inequalities can be deduced. (See Figure 7 for the flowchart of these theorems.) In this section, we will prove Theorem 3.6 and deduce Theorems 3.4 and 3.3. Theorems 4.1 and 4.2 will be stated and proved in the next section. Theorems $3.7,3.5,4.4$, and 4.5 on the lower tail can be deduced by reflecting $X$ to $-X$.

The following inequality is a generalization of the Chernoff inequalities for the binomial distribution:

Theorem 3.3. [Chung and $\mathrm{Lu} 02 \mathrm{~b}$ ] Let $X_{1}, \ldots, X_{n}$ be independent random variables with

$$
\operatorname{Pr}\left(X_{i}=1\right)=p_{i}, \quad \operatorname{Pr}\left(X_{i}=0\right)=1-p_{i} .
$$

For $X=\sum_{i=1}^{n} a_{i} X_{i}$ with $a_{i}>0$, we have $\mathrm{E}(X)=\sum_{i=1}^{n} a_{i} p_{i}$, and we define $\nu=\sum_{i=1}^{n} a_{i}^{2} p_{i}$. Then, we have

$$
\begin{aligned}
& \operatorname{Pr}(X \leq \mathrm{E}(X)-\lambda) \leq e^{-\lambda^{2} / 2 \nu} \\
& \operatorname{Pr}(X \geq \mathrm{E}(X)+\lambda) \leq e^{-\frac{\lambda^{2}}{2(\nu+a \lambda / 3)}}
\end{aligned}
$$

where $a=\max \left\{a_{1}, a_{2}, \ldots, a_{n}\right\}$. 


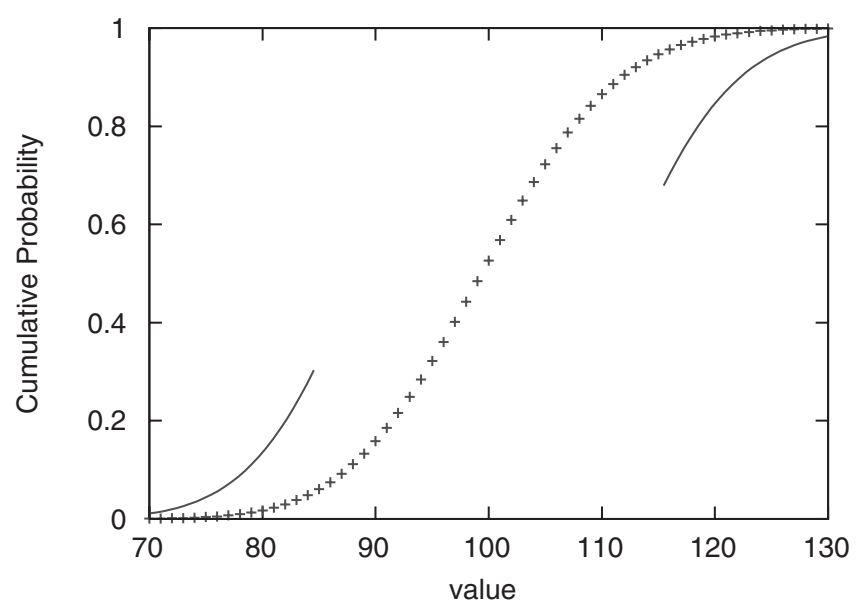

Figure 8. Chernoff inequalities.

To compare inequalities (3.1) to (3.2), we consider an example in Figure 8. The cumulative distribution is the function $\operatorname{Pr}(X>x)$. The dotted curve in Figure 8 illustrates the cumulative distribution of the binomial distribution $B(1000,0.1)$, with the value ranging from 0 to 1 as $x$ goes from $-\infty$ to $\infty$. The solid curve at the lower-left corner is the bound $e^{-\lambda^{2} / 2 \nu}$ for the lower tail. The solid curve at the upper-right corner is the bound $1-e^{-\frac{\lambda^{2}}{2(\nu+a \lambda / 3)}}$ for the upper tail.

The inequality (3.2) in the Theorem 3.3 is a corollary of the following general concentration inequality (also see Theorem 2.7 in the survey paper by McDiarmid [McDiarmid 98]).

Theorem 3.4. [McDiarmid 98] Let $X_{i}(1 \leq i \leq n)$ be independent random variables satisfying $X_{i} \leq \mathrm{E}\left(X_{i}\right)+M$, for $1 \leq i \leq n$. We consider the sum $X=\sum_{i=1}^{n} X_{i}$ with expectation $\mathrm{E}(X)=\sum_{i=1}^{n} \mathrm{E}\left(X_{i}\right)$ and variance $\operatorname{Var}(X)=\sum_{i=1}^{n} \operatorname{Var}\left(X_{i}\right)$. Then, we have

$$
\operatorname{Pr}(X \geq \mathrm{E}(X)+\lambda) \leq e^{-\frac{\lambda^{2}}{2(\operatorname{Var}(X)+M \lambda / 3)}} .
$$

In the other direction, we have the following inequality.

Theorem 3.5. If $X_{1}, X_{2}, \ldots, X_{n}$ are nonnegative independent random variables, we have the following bounds for the sum $X=\sum_{i=1}^{n} X_{i}$ :

$$
\operatorname{Pr}(X \leq \mathrm{E}(X)-\lambda) \leq e^{-\frac{\lambda^{2}}{2 \sum_{i=1}^{n} \mathrm{E}\left(X_{i}^{2}\right)}} .
$$


A strengthened version of Theorem 3.5 is as follows:

Theorem 3.6. Suppose that the $X_{i}$ are independent random variables satisfying $X_{i} \leq M$, for $1 \leq i \leq n$. Let $X=\sum_{i=1}^{n} X_{i}$ and $\|X\|=\sqrt{\sum_{i=1}^{n} \mathrm{E}\left(X_{i}^{2}\right)}$. Then, we have

$$
\operatorname{Pr}(X \geq \mathrm{E}(X)+\lambda) \leq e^{-\frac{\lambda^{2}}{2\left(\|X\|^{2}+M \lambda / 3\right)}} .
$$

Replacing $X$ by $-X$ in the proof of Theorem 3.6, we have the following theorem for the lower tail.

Theorem 3.7. Let $X_{i}$ be independent random variables satisfying $X_{i} \geq-M$, for $1 \leq i \leq n$. Let $X=\sum_{i=1}^{n} X_{i}$ and $\|X\|=\sqrt{\sum_{i=1}^{n} \mathrm{E}\left(X_{i}^{2}\right)}$. Then, we have

$$
\operatorname{Pr}(X \leq \mathrm{E}(X)-\lambda) \leq e^{-\frac{\lambda^{2}}{2\left(\|X\|^{2}+M \lambda / 3\right)}} .
$$

Before we give the proof of Theorems 3.6, we will first show the implications of Theorems 3.6 and 3.7. Namely, we will show that the other concentration inequalities can be derived from Theorems 3.6 and 3.7 .

Fact 3.8. Theorem 3.6 $\Longrightarrow$ Theorem 3.4.

Proof. Let $X_{i}^{\prime}=X_{i}-\mathrm{E}\left(X_{i}\right)$ and $X^{\prime}=\sum_{i=1}^{n} X_{i}^{\prime}=X-\mathrm{E}(X)$. We have

$$
X_{i}^{\prime} \leq M \quad \text { for } 1 \leq i \leq n .
$$

We also have

$$
\begin{aligned}
\left\|X^{\prime}\right\|^{2} & =\sum_{i=1}^{n} \mathrm{E}\left({X^{\prime}}_{i}^{2}\right) \\
& =\sum_{i=1}^{n} \operatorname{Var}\left(X_{i}\right) \\
& =\operatorname{Var}(X) .
\end{aligned}
$$

Applying Theorem 3.6, we get

$$
\begin{aligned}
\operatorname{Pr}(X \geq \mathrm{E}(X)+\lambda) & =\operatorname{Pr}\left(X^{\prime} \geq \lambda\right) \\
& \leq e^{-\frac{\lambda^{2}}{2\left(\left\|X^{\prime}\right\|^{2}+M \lambda / 3\right)}} \\
& \leq e^{-\frac{\lambda^{2}}{2(\operatorname{Var}(X)+M \lambda / 3)}}
\end{aligned}
$$


Fact 3.9. Theorem 3.7 $\Longrightarrow$ Theorem 3.5.

The proof is straightforward by choosing $M=0$.

Fact 3.10. Theorems 3.4 and $3.5 \Longrightarrow$ Theorem 3.3.

Proof. We define $Y_{i}=a_{i} X_{i}$. Note that

$$
\|X\|^{2}=\sum_{i=1}^{n} \mathrm{E}\left(Y_{i}^{2}\right)=\sum_{i=1}^{n} a_{i}^{2} p_{i}=\nu .
$$

Equation (3.1) follows from Theorem 3.5 since the $Y_{i}$ are nonnegatives.

For the other direction, we have

$$
Y_{i} \leq a_{i} \leq a \leq \mathrm{E}\left(Y_{i}\right)+a
$$

Equation (3.2) follows from Theorem 3.4.

Fact 3.II. Theorem 3.6 and Theorem 3.7 $\Longrightarrow$ Theorem 3.1.

The proof is by choosing $Y=X-\mathrm{E}(X)$ and $M=1$ and applying Theorems 3.6 and 3.7 to $Y$.

Fact 3.12. Theorem 3.3 $\Longrightarrow$ Theorem 3.2.

The proof follows by choosing $a_{1}=a_{2}=\cdots=a_{n}=1$.

Finally, we give the complete proof of Theorem 3.6 and thus finish the proofs for all the theorems in this section on Chernoff inequalities.

Proof of Theorem 3.6. We consider

$$
\mathrm{E}\left(e^{t X}\right)=\mathrm{E}\left(e^{t \sum_{i} X_{i}}\right)=\prod_{i=1}^{n} \mathrm{E}\left(e^{t X_{i}}\right),
$$

since the $X_{i}$ are independent.

We define

$$
g(y)=2 \sum_{k=2}^{\infty} \frac{y^{k-2}}{k !}=\frac{2\left(e^{y}-1-y\right)}{y^{2}}
$$

and use the following facts about $g$ :

- $g(0)=1$. 
- $g(y) \leq 1$, for $y<0$.

- $g(y)$ is monotone increasing, for $y \geq 0$.

- For $y<3$, we have

$$
g(y)=2 \sum_{k=2}^{\infty} \frac{y^{k-2}}{k !} \leq \sum_{k=2}^{\infty} \frac{y^{k-2}}{3^{k-2}}=\frac{1}{1-y / 3},
$$

since $k ! \geq 2 \cdot 3^{k-2}$.

Then we have, for $k \geq 2$,

$$
\begin{aligned}
\mathrm{E}\left(e^{t X}\right) & =\prod_{i=1}^{n} \mathrm{E}\left(e^{t X_{i}}\right) \\
& =\prod_{i=1}^{n} \mathrm{E}\left(\sum_{k=0}^{\infty} \frac{t^{k} X_{i}^{k}}{k !}\right) \\
& =\prod_{i=1}^{n} \mathrm{E}\left(1+t \mathrm{E}\left(X_{i}\right)+\frac{1}{2} t^{2} X_{i}^{2} g\left(t X_{i}\right)\right) \\
& \leq \prod_{i=1}^{n}\left(1+t \mathrm{E}\left(X_{i}\right)+\frac{1}{2} t^{2} \mathrm{E}\left(X_{i}^{2}\right) g(t M)\right) \\
& \leq \prod_{i=1}^{n} e^{t \mathrm{E}\left(X_{i}\right)+\frac{1}{2} t^{2} \mathrm{E}\left(X_{i}^{2}\right) g(t M)} \\
& =e^{t \mathrm{E}(X)+\frac{1}{2} t^{2} g(t M) \sum_{i=1}^{n} \mathrm{E}\left(X_{i}^{2}\right)} \\
& =e^{t \mathrm{E}(X)+\frac{1}{2} t^{2} g(t M)\|X\|^{2}} .
\end{aligned}
$$

Hence, for $t$ satisfying $t M<3$, we have

$$
\begin{aligned}
\operatorname{Pr}(X \geq \mathrm{E}(X)+\lambda) & =\operatorname{Pr}\left(e^{t X} \geq e^{t \mathrm{E}(X)+t \lambda}\right) \\
& \leq e^{-t \mathrm{E}(X)-t \lambda} \mathrm{E}\left(e^{t X}\right) \\
& \leq e^{-t \lambda+\frac{1}{2} t^{2} g(t M)\|X\|^{2}} \\
& \leq e^{-t \lambda+\frac{1}{2} t^{2}\|X\|^{2} \frac{1}{1-t M / 3}} .
\end{aligned}
$$

To minimize the above expression, we choose $t=\frac{\lambda}{\|X\|^{2}+M \lambda / 3}$. Therefore, $t M<$ 3 , and we have

$$
\begin{aligned}
\operatorname{Pr}(X \geq \mathrm{E}(X)+\lambda) & \leq e^{-t \lambda+\frac{1}{2} t^{2}\|X\|^{2} \frac{1}{1-t M / 3}} \\
& =e^{-\frac{\lambda^{2}}{2\left(\|X\|^{2}+M \lambda / 3\right)}}
\end{aligned}
$$

The proof is complete. 


\section{More Concentration Inequalities}

Here we state several variations and extensions of the concentration inequalities in Theorem 3.6. We first consider the upper tail.

Theorem 4.I. Let $X_{i}$ denote independent random variables satisfying $X_{i} \leq \mathrm{E}\left(X_{i}\right)+$ $a_{i}+M$, for $1 \leq i \leq n$. For $X=\sum_{i=1}^{n} X_{i}$, we have

$$
\operatorname{Pr}(X \geq \mathrm{E}(X)+\lambda) \leq e^{-\frac{\lambda^{2}}{2\left(\operatorname{Var}(X)+\sum_{i=1}^{n} a_{i}^{2}+M \lambda / 3\right)}}
$$

Proof. Let $X_{i}^{\prime}=X_{i}-\mathrm{E}\left(X_{i}\right)-a_{i}$ and $X^{\prime}=\sum_{i=1}^{n} X_{i}^{\prime}$. We have

$$
\begin{aligned}
X_{i}^{\prime} \leq M & \text { for } 1 \leq i \leq n . \\
X^{\prime}-\mathrm{E}\left(X^{\prime}\right) & =\sum_{i=1}^{n}\left(X_{i}^{\prime}-\mathrm{E}\left(X_{i}^{\prime}\right)\right) \\
& =\sum_{i=1}^{n}\left(X_{i}^{\prime}+a_{i}\right) \\
& =\sum_{i=1}^{n}\left(X_{i}-\mathrm{E}\left(X_{i}\right)\right) \\
& =X-\mathrm{E}(X) .
\end{aligned}
$$

Thus,

$$
\begin{aligned}
\left\|X^{\prime}\right\|^{2} & =\sum_{i=1}^{n} \mathrm{E}\left(X_{i}^{\prime 2}\right) \\
& =\sum_{i=1}^{n} \mathrm{E}\left(\left(X_{i}-\mathrm{E}\left(X_{i}\right)-a_{i}\right)^{2}\right) \\
& =\sum_{i=1}^{n} \mathrm{E}\left(\left(X_{i}-\mathrm{E}\left(X_{i}\right)\right)^{2}+a_{i}^{2}\right. \\
& =\operatorname{Var}(X)+\sum_{i=1}^{n} a_{i}^{2} .
\end{aligned}
$$

By applying Theorem 3.6, the proof is finished.

Theorem 4.2. Suppose that the $X_{i}$ are independent random variables satisfying $X_{i} \leq \mathrm{E}\left(X_{i}\right)+M_{i}$, for $0 \leq i \leq n$. We order the $X_{i}$ so that the $M_{i}$ are in 
increasing order. Let $X=\sum_{i=1}^{n} X_{i}$. Then, for any $1 \leq k \leq n$, we have

$$
\operatorname{Pr}(X \geq \mathrm{E}(X)+\lambda) \leq e^{-\frac{\lambda^{2}}{2\left(\operatorname{Var}(X)+\sum_{i=k}^{n}\left(_{i}-M_{k}\right)^{2}+M_{k} \lambda / 3\right)}} .
$$

Proof. For fixed $k$, we choose $M=M_{k}$ and

$$
a_{i}= \begin{cases}0 & \text { if } 1 \leq i \leq k \\ M_{i}-M_{k} & \text { if } k \leq i \leq n\end{cases}
$$

We have

$$
\begin{gathered}
X_{i}-\mathrm{E}\left(X_{i}\right) \leq M_{i} \leq a_{i}+M_{k} \quad \text { for } 1 \leq k \leq n, \\
\sum_{i=1}^{n} a_{i}^{2}=\sum_{i=k}^{n}\left(M_{i}-M_{k}\right)^{2} .
\end{gathered}
$$

Using Theorem 4.1, we have

$$
\operatorname{Pr}\left(X_{i} \geq \mathrm{E}(X)+\lambda\right) \leq e^{-\frac{\lambda^{2}}{2\left(\operatorname{Var}(X)+\sum_{i=k}^{n}\left(M_{i}-M_{k}\right)^{2}+M_{k} \lambda / 3\right)}} .
$$

Example 4.3. Let $X_{1}, X_{2}, \ldots, X_{n}$ be independent random variables. For $1 \leq i \leq$ $n-1$, suppose that the $X_{i}$ follow the same distribution with

$$
\operatorname{Pr}\left(X_{i}=0\right)=1-p \quad \text { and } \quad \operatorname{Pr}\left(X_{i}=1\right)=p
$$

and that $X_{n}$ follows the distribution with

$$
\operatorname{Pr}\left(X_{n}=0\right)=1-p \quad \text { and } \quad \operatorname{Pr}\left(X_{n}=\sqrt{n}\right)=p .
$$

Consider the sum $X=\sum_{i=1}^{n} X_{i}$.

We have

$$
\begin{aligned}
\mathrm{E}(X) & =\sum_{i=1}^{n} \mathrm{E}\left(X_{i}\right) \\
& =(n-1) p+\sqrt{n} p \\
\operatorname{Var}(X) & =\sum_{i=1}^{n} \operatorname{Var}\left(X_{i}\right) \\
& =(n-1) p(1-p)+n p(1-p) \\
& =(2 n-1) p(1-p)
\end{aligned}
$$


Apply Theorem 3.4 with $M=(1-p) \sqrt{n}$. We have

$$
\operatorname{Pr}(X \geq \mathrm{E}(X)+\lambda) \leq e^{-\frac{\lambda^{2}}{2((2 n-1) p(1-p)+(1-p) \sqrt{n} \lambda / 3)}}
$$

In particular, for constant $p \in(0,1)$ and $\lambda=\Theta\left(n^{\frac{1}{2}+\epsilon}\right)$, we have

$$
\operatorname{Pr}(X \geq \mathrm{E}(X)+\lambda) \leq e^{-\Theta\left(n^{\epsilon}\right)}
$$

Now we apply Theorem 4.2 with $M_{1}=\ldots=M_{n-1}=(1-p)$ and $M_{n}=$ $\sqrt{n}(1-p)$. Choosing $k=n-1$, we have

$$
\begin{aligned}
\operatorname{Var}(X)+\left(M_{n}-M_{n-1}\right)^{2} & =(2 n-1) p(1-p)+(1-p)^{2}(\sqrt{n}-1)^{2} \\
& \leq(2 n-1) p(1-p)+(1-p)^{2} n \\
& \leq\left(1-p^{2}\right) n
\end{aligned}
$$

Thus,

$$
\operatorname{Pr}\left(X_{i} \geq \mathrm{E}(X)+\lambda\right) \leq e^{-\frac{\lambda^{2}}{2\left(\left(1-p^{2}\right) n+(1-p)^{2} \lambda / 3\right)}} .
$$

For constant $p \in(0,1)$ and $\lambda=\Theta\left(n^{\frac{1}{2}+\epsilon}\right)$, we have

$$
\operatorname{Pr}(X \geq \mathrm{E}(X)+\lambda) \leq e^{-\Theta\left(n^{2 \epsilon}\right)} .
$$

From the above examples, we note that Theorem 4.2 gives a significantly better bound than that in Theorem 3.4 if the random variables $X_{i}$ have very different upper bounds.

For completeness, we also list the corresponding theorems for the lower tails. (These can be derived by replacing $X$ by $-X$.)

Theorem 4.4. Let $X_{i}$ denote independent random variables satisfying $X_{i} \geq \mathrm{E}\left(X_{i}\right)-$ $a_{i}-M$, for $0 \leq i \leq n$. For $X=\sum_{i=1}^{n} X_{i}$, we have

$$
\operatorname{Pr}(X \leq \mathrm{E}(X)-\lambda) \leq e^{-\frac{\lambda^{2}}{2\left(\operatorname{Var}(X)+\sum_{i=1}^{n} a_{i}^{2}+M \lambda / 3\right)}} .
$$

Theorem 4.5. Let $X_{i}$ denote independent random variables satisfying $X_{i} \geq \mathrm{E}\left(X_{i}\right)-$ $M_{i}$, for $0 \leq i \leq n$. We order the $X_{i}$ so that the $M_{i}$ are in increasing order. Let $X=\sum_{i=1}^{n} X_{i}$. Then, for any $1 \leq k \leq n$, we have

$$
\operatorname{Pr}(X \leq \mathrm{E}(X)-\lambda) \leq e^{-\frac{\lambda^{2}}{2\left(\operatorname{Var}(X)+\sum_{i=k}^{n}\left(M_{i}-M_{k}\right)^{2}+M_{k} \lambda / 3\right)}} .
$$


Continuing the above example, we choose $M_{1}=M_{2}=\ldots=M_{n-1}=p$ and $M_{n}=\sqrt{n} p$. We choose $k=n-1$, so we have

$$
\begin{aligned}
\operatorname{Var}(X)+\left(M_{n}-M_{n-1}\right)^{2} & =(2 n-1) p(1-p)+p^{2}(\sqrt{n}-1)^{2} \\
& \leq(2 n-1) p(1-p)+p^{2} n \\
& \leq p(2-p) n .
\end{aligned}
$$

Using Theorem 4.5, we have

$$
\operatorname{Pr}(X \leq \mathrm{E}(X)-\lambda) \leq e^{-\frac{\lambda^{2}}{2\left(p(2-p) n+p^{2} \lambda / 3\right)}} .
$$

For a constant $p \in(0,1)$ and $\lambda=\Theta\left(n^{\frac{1}{2}+\epsilon}\right)$, we have

$$
\operatorname{Pr}(X \leq \mathrm{E}(X)-\lambda) \leq e^{-\Theta\left(n^{2 \epsilon}\right)}
$$

\section{Martingales and Azuma's Inequality}

A martingale is a sequence of random variables $X_{0}, X_{1}, \ldots$ with finite means such that the conditional expectation of $X_{n+1}$ given $X_{0}, X_{1}, \ldots, X_{n}$ is equal to $X_{n}$.

The above definition is given in the classical book of Feller [Feller 71, p. 210]. However, the conditional expectation depends on the random variables under consideration and can be difficult to deal with in various cases. In this survey we will use the following definition, which is concise and basically equivalent for the finite cases.

Suppose that $\Omega$ is a probability space with a probability distribution $p$. Let $\mathcal{F}$ denote a $\sigma$-field on $\Omega$. (A $\sigma$-field on $\Omega$ is a collection of subsets of $\Omega$ that contains $\emptyset$ and $\Omega$ and is closed under unions, intersections, and complementation.) In a $\sigma$-field $\mathcal{F}$ of $\Omega$, the smallest set in $\mathcal{F}$ containing an element $x$ is the intersection of all sets in $\mathcal{F}$ containing $x$. A function $f: \Omega \rightarrow \mathbb{R}$ is said to be $\mathcal{F}$-measurable if $f(x)=f(y)$ for any $y$ in the smallest set containing $x$. (For more terminology on martingales, the reader is referred to [Janson et al. 00].)

If $f: \Omega \rightarrow \mathbb{R}$ is a function, we define the expectation $\mathrm{E}(f)=\mathrm{E}(f(x) \mid x \in \Omega)$ by

$$
\mathrm{E}(f)=\mathrm{E}(f(x) \mid x \in \Omega):=\sum_{x \in \Omega} f(x) p(x) .
$$

If $\mathcal{F}$ is a $\sigma$-field on $\Omega$, we define the conditional expectation $\mathrm{E}(f \mid \mathcal{F}): \Omega \rightarrow \mathbb{R}$ by the formula

$$
\mathrm{E}(f \mid \mathcal{F})(x):=\frac{1}{\sum_{y \in \mathcal{F}(x)} p(y)} \sum_{y \in \mathcal{F}(x)} f(y) p(y),
$$

where $\mathcal{F}(x)$ is the smallest element of $\mathcal{F}$ that contains $x$. 
A filter $\mathbf{F}$ is an increasing chain of $\sigma$-subfields

$$
\{0, \Omega\}=\mathcal{F}_{0} \subset \mathcal{F}_{1} \subset \cdots \subset \mathcal{F}_{n}=\mathcal{F}
$$

A martingale (obtained from) $X$ is associated with a filter $\mathbf{F}$ and a sequence of random variables $X_{0}, X_{1}, \ldots, X_{n}$ satisfying $X_{i}=\mathrm{E}\left(X \mid \mathcal{F}_{i}\right)$ and, in particular, $X_{0}=\mathrm{E}(X)$ and $X_{n}=X$.

Example 5.I. For given independent random variables $Y_{1}, Y_{2}, \ldots, Y_{n}$, we can define a martingale $X=Y_{1}+Y_{2}+\cdots+Y_{n}$ as follows. Let $\mathcal{F}_{i}$ be the $\sigma$-field generated by $Y_{1}, \ldots, Y_{i}$. (In other words, $\mathcal{F}_{i}$ is the minimum $\sigma$-field so that $Y_{1}, \ldots, Y_{i}$ are $\mathcal{F}_{i}$-measurable.) We have a natural filter $\mathbf{F}$ :

$$
\{0, \Omega\}=\mathcal{F}_{0} \subset \mathcal{F}_{1} \subset \cdots \subset \mathcal{F}_{n}=\mathcal{F}
$$

Let $X_{i}=\sum_{j=1}^{i} Y_{j}+\sum_{j=i+1}^{n} \mathrm{E}\left(Y_{j}\right)$. Then, $X_{0}, X_{1}, X_{2}, \ldots, X_{n}$ forms a martingale corresponding to the filter $\mathbf{F}$.

For $\mathbf{c}=\left(c_{1}, c_{2}, \ldots, c_{n}\right)$ a vector with positive entries, the martingale $X$ is said to be $\mathbf{c}$-Lipschitz if

$$
\left|X_{i}-X_{i-1}\right| \leq c_{i}
$$

for $i=1,2, \ldots, n$. The following theorem is a powerful tool for controlling martingales.

Theorem 5.2. (Azuma's inequality.) If a martingale $X$ is c-Lipschitz, then

$$
\operatorname{Pr}(|X-\mathrm{E}(X)| \geq \lambda) \leq 2 e^{-\frac{\lambda^{2}}{2 \sum_{i=1}^{n} c_{i}^{2}}},
$$

where $\mathbf{c}=\left(c_{1}, \ldots, c_{n}\right)$.

Theorem 5.3. Let $X_{1}, X_{2}, \ldots, X_{n}$ be independent random variables satisfying

$$
\left|X_{i}-\mathrm{E}\left(X_{i}\right)\right| \leq c_{i} \quad \text { for } 1 \leq i \leq n
$$

Then, we have the following bound for the sum $X=\sum_{i=1}^{n} X_{i}$ :

$$
\operatorname{Pr}(|X-\mathrm{E}(X)| \geq \lambda) \leq 2 e^{-\frac{\lambda^{2}}{2 \sum_{i=1}^{\lambda^{n}} c_{i}^{2}}}
$$


Proof of Azuma's inequality. For a fixed $t$, we consider the convex function $f(x)=e^{t x}$. For any $|x| \leq c, f(x)$ is below the line segment from $(-c, f(-c))$ to $(c, f(c))$. In other words, we have

$$
e^{t x} \leq \frac{1}{2 c}\left(e^{t c}-e^{-t c}\right) x+\frac{1}{2}\left(e^{t c}+e^{-t c}\right) .
$$

Therefore, we can write

$$
\begin{aligned}
\mathrm{E}\left(e^{t\left(X_{i}-X_{i-1}\right)} \mid \mathcal{F}_{i-1}\right) & \\
& \leq \mathrm{E}\left(\frac{1}{2 c_{i}}\left(e^{t c_{i}}-e^{-t c_{i}}\right)\left(X_{i}-X_{i-1}\right)+\frac{1}{2}\left(e^{t c_{i}}+e^{-t c_{i}}\right) \mid \mathcal{F}_{i-1}\right) \\
& =\frac{1}{2}\left(e^{t c_{i}}+e^{-t c_{i}}\right) \\
& \leq e^{t^{2} c_{i}^{2} / 2}
\end{aligned}
$$

Here we apply the conditions $\mathrm{E}\left(X_{i}-X_{i-1} \mid \mathcal{F}_{i-1}\right)=0$ and $\left|X_{i}-X_{i-1}\right| \leq c_{i}$.

Hence,

$$
\mathrm{E}\left(e^{t X_{i}} \mid \mathcal{F}_{i-1}\right) \leq e^{t^{2} c_{i}^{2} / 2} e^{t X_{i-1}}
$$

Inductively, we have

$$
\begin{aligned}
& \mathrm{E}\left(e^{t X}\right)=\mathrm{E}\left(\mathrm{E}\left(e^{t X_{n}} \mid \mathcal{F}_{n-1}\right)\right) \\
& \leq e^{t^{2} c_{n}^{2} / 2} \mathrm{E}\left(e^{t X_{n-1}}\right) \\
& \vdots \\
& \leq \prod_{i=1}^{n} e^{t^{2} c_{i}^{2} / 2} \mathrm{E}\left(e^{t X_{0}}\right) \\
&=e^{\frac{1}{2} t^{2} \sum_{i=1}^{n} c_{i}^{2}} e^{t \mathrm{E}(X)} .
\end{aligned}
$$

Therefore,

$$
\begin{aligned}
\operatorname{Pr}(X \geq \mathrm{E}(X)+\lambda) & =\operatorname{Pr}\left(e^{t(X-\mathrm{E}(X))} \geq e^{t \lambda}\right) \\
& \leq e^{-t \lambda} \mathrm{E}\left(e^{t(X-\mathrm{E}(X))}\right) \\
& \leq e^{-t \lambda} e^{\frac{1}{2} t^{2} \sum_{i=1}^{n} c_{i}^{2}} \\
& =e^{-t \lambda+\frac{1}{2} t^{2} \sum_{i=1}^{n} c_{i}^{2}}
\end{aligned}
$$

We choose $t=\frac{\lambda}{\sum_{i=1}^{n} c_{i}^{2}}$ (in order to minimize the previous expression). We have

$$
\begin{aligned}
\operatorname{Pr}(X \geq \mathrm{E}(X)+\lambda) & \leq e^{-t \lambda+\frac{1}{2} t^{2} \sum_{i=1}^{n} c_{i}^{2}} \\
& =e^{-\frac{\lambda^{2}}{2 \sum_{i=1}^{n} c_{i}^{2}}}
\end{aligned}
$$


To derive a similar lower bound, we consider $-X_{i}$ instead of $X_{i}$ in the preceding proof. Then, we obtain the following bound for the lower tail:

$$
\operatorname{Pr}(X \leq \mathrm{E}(X)-\lambda) \leq e^{-\frac{\lambda^{2}}{2 \sum_{i=1}^{n} c_{i}^{2}}}
$$

\section{General Martingale Inequalities}

Many problems that can be set up as a martingale do not satisfy the Lipschitz condition. It is desirable to be able to use tools similar to the Azuma inequality in such cases. In this section, we will first state and then prove several extensions of the Azuma inequality (see Figure 9).

Our starting point is the following well-known concentration inequality (see [McDiarmid 98]):

Theorem 6.I. Let $X$ be the martingale associated with a filter $\mathbf{F}$ satisfying

1. $\operatorname{Var}\left(X_{i} \mid \mathcal{F}_{i-1}\right) \leq \sigma_{i}^{2}$, for $1 \leq i \leq n$;

2. $\left|X_{i}-X_{i-1}\right| \leq M$, for $1 \leq i \leq n$.

Then, we have

$$
\operatorname{Pr}(X-\mathrm{E}(X) \geq \lambda) \leq e^{-\frac{\lambda^{2}}{2\left(\sum_{i=1}^{n} \sigma_{i}^{2}+M \lambda / 3\right)}}
$$

Since the sum of independent random variables can be viewed as a martingale (see Example 5.1), Theorem 6.1 implies Theorem 3.4. In a similar way, the following theorem is associated with Theorem 4.1.

Upper tails

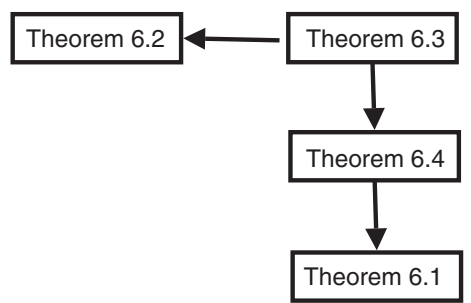

Lower tails

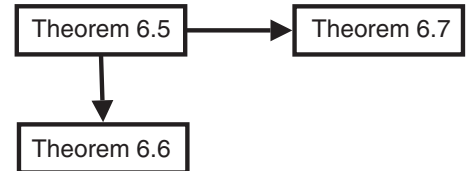

Theorem 6.6

Figure 9. The flowchart for theorems on martingales. 
Theorem 6.2. Let $X$ be the martingale associated with a filter $\mathbf{F}$ satisfying

1. $\operatorname{Var}\left(X_{i} \mid \mathcal{F}_{i-1}\right) \leq \sigma_{i}^{2}$, for $1 \leq i \leq n$;

2. $X_{i}-X_{i-1} \leq M_{i}$, for $1 \leq i \leq n$.

Then, we have

$$
\operatorname{Pr}(X-\mathrm{E}(X) \geq \lambda) \leq e^{-\frac{\lambda^{2}}{2 \sum_{i=1}^{n}\left(\sigma_{i}^{2}+M_{i}^{2}\right)}} .
$$

Theorem 6.2 can be further generalized:

Theorem 6.3. Let $X$ be the martingale associated with a filter $\mathbf{F}$ satisfying

1. $\operatorname{Var}\left(X_{i} \mid \mathcal{F}_{i-1}\right) \leq \sigma_{i}^{2}$, for $1 \leq i \leq n$;

2. $X_{i}-X_{i-1} \leq a_{i}+M$, for $1 \leq i \leq n$.

Then, we have

$$
\operatorname{Pr}(X-\mathrm{E}(X) \geq \lambda) \leq e^{-\frac{\lambda^{2}}{2\left(\sum_{i=1}^{n}\left(\sigma_{i}^{2}+a_{i}^{2}\right)+M \lambda / 3\right)}} .
$$

Theorem 6.3 implies Theorem 6.1 by choosing $a_{1}=a_{2}=\cdots=a_{n}=0$.

We also have the following theorem corresponding to Theorem 4.2.

Theorem 6.4. Let $X$ be the martingale associated with a filter $\mathbf{F}$ satisfying

1. $\operatorname{Var}\left(X_{i} \mid \mathcal{F}_{i-1}\right) \leq \sigma_{i}^{2}$, for $1 \leq i \leq n$;

2. $X_{i}-X_{i-1} \leq M_{i}$, for $1 \leq i \leq n$.

Then, for any $M$, we have

$$
\operatorname{Pr}(X-\mathrm{E}(X) \geq \lambda) \leq e^{-\frac{\lambda^{2}}{2\left(\sum_{i=1}^{n} \sigma_{i}^{2}+\sum_{M_{i}}>M^{\left.\left(M_{i}-M\right)^{2}+M \lambda / 3\right)}\right.}} .
$$

Theorem 6.3 implies Theorem 6.4 by choosing

$$
a_{i}= \begin{cases}0 & \text { if } M_{i} \leq M \\ M_{i}-M & \text { if } M_{i} \geq M\end{cases}
$$

It suffices to prove Theorem 6.3 so that all the above stated theorems hold.

Proof of Theorem 6.3. Recall that $g(y)=2 \sum_{k=2}^{\infty} \frac{y^{k-2}}{k !}$ satisfies the following properties: 
- $g(y) \leq 1$, for $y<0$.

- $\lim _{y \rightarrow 0} g(y)=1$.

- $g(y)$ is monotone increasing, for $y \geq 0$.

- When $b<3$, we have $g(b) \leq \frac{1}{1-b / 3}$.

Since $\mathrm{E}\left(X_{i} \mid \mathcal{F}_{i-1}\right)=X_{i-1}$ and $X_{i}-X_{i-1}-a_{i} \leq M$, we have

$$
\begin{aligned}
\mathrm{E}\left(e^{t\left(X_{i}-X_{i-1}-a_{i}\right)} \mid \mathcal{F}_{i-1}\right) & =\mathrm{E}\left(\sum_{k=0}^{\infty} \frac{t^{k}}{k !}\left(X_{i}-X_{i-1}-a_{i}\right)^{k} \mid \mathcal{F}_{i-1}\right) \\
& =1-t a_{i}+\mathrm{E}\left(\sum_{k=2}^{\infty} \frac{t^{k}}{k !}\left(X_{i}-X_{i-1}-a_{i}\right)^{k} \mid \mathcal{F}_{i-1}\right) \\
& \leq 1-t a_{i}+\mathrm{E}\left(\frac{t^{2}}{2}\left(X_{i}-X_{i-1}-a_{i}\right)^{2} g(t M) \mid \mathcal{F}_{i-1}\right) \\
& =1-t a_{i}+\frac{t^{2}}{2} g(t M) \mathrm{E}\left(\left(X_{i}-X_{i-1}-a_{i}\right)^{2} \mid \mathcal{F}_{i-1}\right) \\
& =1-t a_{i}+\frac{t^{2}}{2} g(t M)\left(\mathrm{E}\left(\left(X_{i}-X_{i-1}\right)^{2} \mid \mathcal{F}_{i-1}\right)+a_{i}^{2}\right) \\
& \leq 1-t a_{i}+\frac{t^{2}}{2} g(t M)\left(\sigma_{i}^{2}+a_{i}^{2}\right) \\
& \leq e^{-t a_{i}+\frac{t^{2}}{2} g(t M)\left(\sigma_{i}^{2}+a_{i}^{2}\right)} .
\end{aligned}
$$

Thus,

$$
\begin{aligned}
\mathrm{E}\left(e^{t X_{i}} \mid \mathcal{F}_{i-1}\right) & =\mathrm{E}\left(e^{t\left(X_{i}-X_{i-1}-a_{i}\right)} \mid \mathcal{F}_{i-1}\right) e^{t X_{i-1}+t a_{i}} \\
& \leq e^{-t a_{i}+\frac{t^{2}}{2} g(t M)\left(\sigma_{i}^{2}+a_{i}^{2}\right)} e^{t X_{i-1}+t a_{i}} \\
& =e^{\frac{t^{2}}{2} g(t M)\left(\sigma_{i}^{2}+a_{i}^{2}\right)} e^{t X_{i-1}}
\end{aligned}
$$

Inductively, we have

$$
\begin{aligned}
& \mathrm{E}\left(e^{t X}\right)=\mathrm{E}\left(\mathrm{E}\left(e^{t X_{n}} \mid \mathcal{F}_{n-1}\right)\right) \\
& \leq e^{\frac{t^{2}}{2} g(t M)\left(\sigma_{n}^{2}+a_{n}^{2}\right)} \mathrm{E}\left(e^{t X_{n-1}}\right) \\
& \vdots \\
& \leq \prod_{i=1}^{n} e^{\frac{t^{2}}{2} g(t M)\left(\sigma_{i}^{2}+a_{i}^{2}\right)} \mathrm{E}\left(e^{t X_{0}}\right) \\
&=e^{\frac{1}{2} t^{2} g(t M) \sum_{i=1}^{n}\left(\sigma_{i}^{2}+a_{i}^{2}\right)} e^{t \mathrm{E}(X)} .
\end{aligned}
$$


Then for $t$ satisfying $t M<3$, we have

$$
\begin{aligned}
\operatorname{Pr}(X \geq \mathrm{E}(X)+\lambda) & =\operatorname{Pr}\left(e^{t X} \geq e^{t \mathrm{E}(X)+t \lambda}\right) \\
& \leq e^{-t \mathrm{E}(X)-t \lambda} \mathrm{E}\left(e^{t X}\right) \\
& \leq e^{-t \lambda} e^{\frac{1}{2} t^{2} g(t M) \sum_{i=1}^{n}\left(\sigma_{i}^{2}+a_{i}^{2}\right)} \\
& =e^{-t \lambda+\frac{1}{2} t^{2} g(t M) \sum_{i=1}^{n}\left(\sigma_{i}^{2}+a_{i}^{2}\right)} \\
& \leq e^{-t \lambda+\frac{1}{2} \frac{t^{2}}{1-t M / 3} \sum_{i=1}^{n}\left(\sigma_{i}^{2}+a_{i}^{2}\right)}
\end{aligned}
$$

We choose $t=\frac{\lambda}{\sum_{i=1}^{n}\left(\sigma_{i}^{2}+a_{i}^{2}\right)+M \lambda / 3}$. Clearly $t M<3$ and

$$
\begin{aligned}
\operatorname{Pr}(X \geq \mathrm{E}(X)+\lambda) & \leq e^{-t \lambda+\frac{1}{2} \frac{t^{2}}{1-t M / 3} \sum_{i=1}^{n}\left(\sigma_{i}^{2}+a_{i}^{2}\right)} \\
& =e^{-\frac{\lambda^{2}}{2\left(\sum_{i=1}^{n}\left(\sigma_{i}^{2}+a_{i}^{2}\right)+M \lambda / 3\right)}} .
\end{aligned}
$$

The proof of the theorem is complete.

For completeness, we state the following theorems for the lower tails. The proofs are almost identical and will be omitted.

Theorem 6.5. Let $X$ be the martingale associated with a filter $\mathbf{F}$ satisfying

1. $\operatorname{Var}\left(X_{i} \mid \mathcal{F}_{i-1}\right) \leq \sigma_{i}^{2}$, for $1 \leq i \leq n$;

2. $X_{i-1}-X_{i} \leq a_{i}+M$, for $1 \leq i \leq n$.

Then, we have

$$
\operatorname{Pr}(X-\mathrm{E}(X) \leq-\lambda) \leq e^{-\frac{\lambda^{2}}{2\left(\sum_{i=1}^{n}\left(\sigma_{i}^{2}+a_{i}^{2}\right)+M \lambda / 3\right)}} .
$$

Theorem 6.6. Let $X$ be the martingale associated with a filter $\mathbf{F}$ satisfying

1. $\operatorname{Var}\left(X_{i} \mid \mathcal{F}_{i-1}\right) \leq \sigma_{i}^{2}$, for $1 \leq i \leq n$;

2. $X_{i-1}-X_{i} \leq M_{i}$, for $1 \leq i \leq n$.

Then, we have

$$
\operatorname{Pr}(X-\mathrm{E}(X) \leq-\lambda) \leq e^{-\frac{\lambda^{2}}{2 \sum_{i=1}^{n}\left(\sigma_{i}^{2}+M_{i}^{2}\right)}}
$$


Theorem 6.7. Let $X$ be the martingale associated with a filter $\mathbf{F}$ satisfying

1. $\operatorname{Var}\left(X_{i} \mid \mathcal{F}_{i-1}\right) \leq \sigma_{i}^{2}$, for $1 \leq i \leq n$;

2. $X_{i-1}-X_{i} \leq M_{i}$, for $1 \leq i \leq n$.

Then, for any $M$, we have

$$
\operatorname{Pr}(X-\mathrm{E}(X) \leq-\lambda) \leq e^{-\frac{\lambda^{2}}{2\left(\sum_{i=1}^{n} \sigma_{i}^{2}+\sum_{\left.\left.M_{i}>M^{(} M_{i}-M\right)^{2}+M \lambda / 3\right)}\right.}} .
$$

\section{Supermartingales and Submartingales}

In this section, we consider further-strengthened versions of the martingale inequalities that have been mentioned so far. Instead of a fixed upper bound for the variance, we will assume that the variance $\operatorname{Var}\left(X_{i} \mid \mathcal{F}_{i-1}\right)$ is upper bounded by a linear function of $X_{i-1}$. Here we assume this linear function is nonnegative for all values that $X_{i-1}$ takes. We first need some terminology.

For a filter $\mathbf{F}$,

$$
\{\emptyset, \Omega\}=\mathcal{F}_{0} \subset \mathcal{F}_{1} \subset \cdots \subset \mathcal{F}_{n}=\mathcal{F}
$$

a sequence of random variables $X_{0}, X_{1}, \ldots, X_{n}$ is called a submartingale if $X_{i}$ is $\mathcal{F}_{i}$-measurable (i.e., $X_{i}(a)=X_{i}(b)$ if all elements of $\mathcal{F}_{i}$ that contain $a$ also contain $b$ and vice versa) and $\mathrm{E}\left(X_{i} \mid \mathcal{F}_{i-1}\right) \leq X_{i-1}$, for $1 \leq i \leq n$.

A sequence of random variables $X_{0}, X_{1}, \ldots, X_{n}$ is said to be a supermartingale if $X_{i}$ is $\mathcal{F}_{i}$-measurable and $\mathrm{E}\left(X_{i} \mid \mathcal{F}_{i-1}\right) \geq X_{i-1}$, for $1 \leq i \leq n$.

To avoid repetition, we will first state a number of useful inequalities for submartingales and supermartingales. Then, we will give the proof for the general inequalities in Theorem 7.3 for submartingales and in Theorem 7.5 for supermartingales. Furthermore, we will show that all the stated theorems follow from Theorems 7.3 and 7.5. (See Figure 10.) Note that the inequalities for submartingale and supermartingale are not quite symmetric.

Submartingale

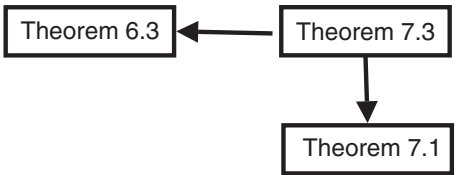

\section{Supermartingale}

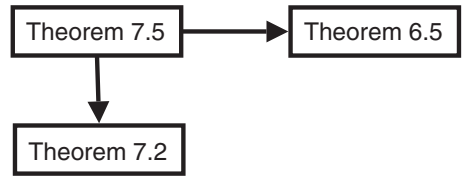

Figure 10. The flowchart for theorems on submartingales and supermartingales. 
Theorem 7.I. Suppose that a submartingale $X$, associated with a filter $\mathbf{F}$, satisfies

$$
\operatorname{Var}\left(X_{i} \mid \mathcal{F}_{i-1}\right) \leq \phi_{i} X_{i-1}
$$

and

$$
X_{i}-\mathrm{E}\left(X_{i} \mid \mathcal{F}_{i-1}\right) \leq M
$$

for $1 \leq i \leq n$. Then, we have

$$
\operatorname{Pr}\left(X_{n} \geq X_{0}+\lambda\right) \leq e^{-\frac{\lambda^{2}}{2\left(\left(X_{0}+\lambda\right)\left(\sum_{i=1}^{n} \phi_{i}\right)+M \lambda / 3\right)}} .
$$

Theorem 7.2. Suppose that a supermartingale $X$, associated with a filter $\mathbf{F}$, satisfies, for $1 \leq i \leq n$,

$$
\operatorname{Var}\left(X_{i} \mid \mathcal{F}_{i-1}\right) \leq \phi_{i} X_{i-1}
$$

and

$$
\mathrm{E}\left(X_{i} \mid \mathcal{F}_{i-1}\right)-X_{i} \leq M
$$

Then, we have

$$
\operatorname{Pr}\left(X_{n} \leq X_{0}-\lambda\right) \leq e^{-\frac{\lambda^{2}}{2\left(X_{0}\left(\sum_{i=1}^{n} \phi_{i}\right)+M \lambda / 3\right)}}
$$

for any $\lambda \leq X_{0}$.

Theorem 7.3. Suppose that a submartingale $X$, associated with a filter $\mathbf{F}$, satisfies

$$
\operatorname{Var}\left(X_{i} \mid \mathcal{F}_{i-1}\right) \leq \sigma_{i}^{2}+\phi_{i} X_{i-1}
$$

and

$$
X_{i}-\mathrm{E}\left(X_{i} \mid \mathcal{F}_{i-1}\right) \leq a_{i}+M
$$

for $1 \leq i \leq n$. Here, $\sigma_{i}, a_{i}, \phi_{i}$, and $M$ are nonnegative constants. Then, we have

$$
\operatorname{Pr}\left(X_{n} \geq X_{0}+\lambda\right) \leq e^{-\frac{\lambda^{2}}{2\left(\sum_{i=1}^{n}\left(\sigma_{i}^{2}+a_{i}^{2}\right)+\left(X_{0}+\lambda\right)\left(\sum_{i=1}^{n} \phi_{i}\right)+M \lambda / 3\right)}} .
$$

Remark 7.4. Theorem 7.3 implies Theorem 7.1 by setting all $\sigma_{i}$ and $a_{i}$ to zero. Theorem 7.3 also implies Theorem 6.3 by choosing $\phi_{1}=\cdots=\phi_{n}=0$.

The theorem for a supermartingale is slightly different due to the asymmetry of the condition on the variance.

Theorem 7.5. Suppose that a supermartingale $X$, associated with a filter $\mathbf{F}$, satisfies, for $1 \leq i \leq n$,

$$
\operatorname{Var}\left(X_{i} \mid \mathcal{F}_{i-1}\right) \leq \sigma_{i}^{2}+\phi_{i} X_{i-1}
$$


and

$$
\mathrm{E}\left(X_{i} \mid \mathcal{F}_{i-1}\right)-X_{i} \leq a_{i}+M,
$$

where $M, a_{i}, \sigma_{i}$, and $\phi_{i}$ are nonnegative constants. Then, we have

$$
\operatorname{Pr}\left(X_{n} \leq X_{0}-\lambda\right) \leq e^{-\frac{\lambda^{2}}{2\left(\sum_{i=1}^{n}\left(\sigma_{i}^{2}+a_{i}^{2}\right)+X_{0}\left(\sum_{i=1}^{n} \phi_{i}\right)+M \lambda / 3\right)}},
$$

for any $\lambda \leq 2 X_{0}+\frac{\sum_{i=1}^{n}\left(\sigma_{i}^{2}+a_{i}^{2}\right)}{\sum_{i=1}^{n} \phi_{i}}$.

Remark 7.6. Theorem 7.5 implies Theorem 7.2 by setting all $\sigma_{i}$ and $a_{i}$ to zero.

Theorem 7.5 also implies Theorem 6.5 by choosing $\phi_{1}=\cdots=\phi_{n}=0$.

Proof of Theorem 7.3. For a positive $t$ (to be chosen later), we consider

$$
\begin{aligned}
\mathrm{E}\left(e^{t X_{i}} \mid \mathcal{F}_{i-1}\right) & =e^{t \mathrm{E}\left(X_{i} \mid \mathcal{F}_{i-1}\right)+t a_{i}} \mathrm{E}\left(e^{t\left(X_{i}-\mathrm{E}\left(X_{i} \mid \mathcal{F}_{i-1}\right)-a_{i}\right)} \mid \mathcal{F}_{i-1}\right) \\
& =e^{t \mathrm{E}\left(X_{i} \mid \mathcal{F}_{i-1}\right)+t a_{i}} \sum_{k=0}^{\infty} \frac{t^{k}}{k !} \mathrm{E}\left(\left(X_{i}-\mathrm{E}\left(X_{i} \mid \mathcal{F}_{i-1}\right)-a_{i}\right)^{k} \mid \mathcal{F}_{i-1}\right) \\
& \leq e^{t \mathrm{E}\left(X_{i} \mid \mathcal{F}_{i-1}\right)+\sum_{k=2}^{\infty} \frac{t^{k}}{k !} \mathrm{E}\left(\left(X_{i}-\mathrm{E}\left(X_{i} \mid \mathcal{F}_{i-1}\right)-a_{i}\right)^{k} \mid \mathcal{F}_{i-1}\right)}
\end{aligned}
$$

Recall that $g(y)=2 \sum_{k=2}^{\infty} \frac{y^{k-2}}{k !}$ satisfies

$$
g(y) \leq g(b)<\frac{1}{1-b / 3}
$$

for all $y \leq b$ and $0 \leq b \leq 3$.

Since $X_{i}-\mathrm{E}\left(X_{i} \mid \mathcal{F}_{i-1}\right)-a_{i} \leq M$, we have

$$
\begin{aligned}
\sum_{k=2}^{\infty} \frac{t^{k}}{k !} \mathrm{E}\left(\left(X_{i}-\mathrm{E}\left(X_{i} \mid \mathcal{F}_{i-1}\right)-a_{i}\right)^{k} \mid \mathcal{F}_{i-1}\right) \\
\leq \frac{g(t M)}{2} t^{2} \mathrm{E}\left(\left(X_{i}-\mathrm{E}\left(X_{i} \mid \mathcal{F}_{i-1}\right)-a_{i}\right)^{2} \mid \mathcal{F}_{i-1}\right) \\
=\frac{g(t M)}{2} t^{2}\left(\operatorname{Var}\left(X_{i} \mid \mathcal{F}_{i-1}\right)+a_{i}^{2}\right) \\
\leq \frac{g(t M)}{2} t^{2}\left(\sigma_{i}^{2}+\phi_{i} X_{i-1}+a_{i}^{2}\right) .
\end{aligned}
$$

Since $\mathrm{E}\left(X_{i} \mid \mathcal{F}_{i-1}\right) \leq X_{i-1}$, we have

$$
\begin{aligned}
\mathrm{E}\left(e^{t X_{i}} \mid \mathcal{F}_{i-1}\right) & \leq e^{t \mathrm{E}\left(X_{i} \mid \mathcal{F}_{i-1}\right)+\sum_{k=2}^{\infty} \frac{t^{k}}{k !} \mathrm{E}\left(\left(X_{i}-\mathrm{E}\left(X_{i} \mid \mathcal{F}_{i-1}-\right)-a_{i}\right)^{k} \mid \mathcal{F}_{i-1}\right)} \\
& \leq e^{t X_{i-1}+\frac{g(t M)}{2} t^{2}\left(\sigma_{i}^{2}+\phi_{i} X_{i-1}+a_{i}^{2}\right)} \\
& =e^{\left(t+\frac{g(t M)}{2} \phi_{i} t^{2}\right) X_{i-1}} e^{\frac{t^{2}}{2} g(t M)\left(\sigma_{i}^{2}+a_{i}^{2}\right)}
\end{aligned}
$$


We define $t_{i} \geq 0$ for $0<i \leq n$, satisfying

$$
t_{i-1}=t_{i}+\frac{g\left(t_{0} M\right)}{2} \phi_{i} t_{i}^{2}
$$

while $t_{0}$ will be chosen later. Then,

$$
t_{n} \leq t_{n-1} \leq \cdots \leq t_{0}
$$

and

$$
\begin{aligned}
\mathrm{E}\left(e^{t_{i} X_{i}} \mid \mathcal{F}_{i-1}\right) & \leq e^{\left(t_{i}+\frac{g\left(t_{i} M\right)}{2} \phi_{i} t_{i}^{2}\right) X_{i-1}} e^{\frac{t_{i}^{2}}{2} g\left(t_{i} M\right)\left(\sigma_{i}^{2}+a_{i}^{2}\right)} \\
& \leq e^{\left(t_{i}+\frac{g\left(t_{0} M\right)}{2} t_{i}^{2} \phi_{i}\right) X_{i-1}} e^{\frac{t_{i}^{2}}{2} g\left(t_{i} M\right)\left(\sigma_{i}^{2}+a_{i}^{2}\right)} \\
& =e^{t_{i-1} X_{i-1}} e^{\frac{t_{i}^{2}}{2} g\left(t_{i} M\right)\left(\sigma_{i}^{2}+a_{i}^{2}\right)}
\end{aligned}
$$

since $g(y)$ is increasing for $y>0$.

By Markov's inequality, we have

$$
\begin{aligned}
\operatorname{Pr}\left(X_{n} \geq X_{0}+\lambda\right) & \leq e^{-t_{n}\left(X_{0}+\lambda\right)} \mathrm{E}\left(e^{t_{n} X_{n}}\right) \\
& =e^{-t_{n}\left(X_{0}+\lambda\right)} \mathrm{E}\left(\mathrm{E}\left(e^{t_{n} X_{n}} \mid \mathcal{F}_{n-1}\right)\right) \\
& \leq e^{-t_{n}\left(X_{0}+\lambda\right)} \mathrm{E}\left(e^{t_{n-1} X_{n-1}}\right) e^{\frac{t_{i}^{2}}{2} g\left(t_{i} M\right)\left(\sigma_{i}^{2}+a_{i}^{2}\right)} \\
& \vdots \\
& \leq e^{-t_{n}\left(X_{0}+\lambda\right)} \mathrm{E}\left(e^{t_{0} X_{0}}\right) e^{\sum_{i=1}^{n} \frac{t_{i}^{2}}{2} g\left(t_{i} M\right)\left(\sigma_{i}^{2}+a_{i}^{2}\right)} \\
& \leq e^{-t_{n}\left(X_{0}+\lambda\right)+t_{0} X_{0}+\frac{t_{0}^{2}}{2} g\left(t_{0} M\right) \sum_{i=1}^{n}\left(\sigma_{i}^{2}+a_{i}^{2}\right)} .
\end{aligned}
$$

Note that

$$
\begin{aligned}
t_{n} & =t_{0}-\sum_{i=1}^{n}\left(t_{i-1}-t_{i}\right) \\
& =t_{0}-\sum_{i=1}^{n} \frac{g\left(t_{0} M\right)}{2} \phi_{i} t_{i}^{2} \\
& \geq t_{0}-\frac{g\left(t_{0} M\right)}{2} t_{0}^{2} \sum_{i=1}^{n} \phi_{i} .
\end{aligned}
$$

Hence,

$$
\begin{aligned}
\operatorname{Pr}\left(X_{n} \geq X_{0}+\lambda\right) & \leq e^{-t_{n}\left(X_{0}+\lambda\right)+t_{0} X_{0}+\frac{t_{0}^{2}}{2} g\left(t_{0} M\right) \sum_{i=1}^{n}\left(\sigma_{i}^{2}+a_{i}^{2}\right)} \\
& \leq e^{-\left(t_{0}-\frac{g\left(t_{0} M\right)}{2} t_{0}^{2} \sum_{i=1}^{n} \phi_{i}\right)\left(X_{0}+\lambda\right)+t_{0} X_{0}+\frac{t_{0}^{2}}{2} g\left(t_{0} M\right) \sum_{i=1}^{n}\left(\sigma_{i}^{2}+a_{i}^{2}\right)} \\
& =e^{-t_{0} \lambda+\frac{g\left(t_{0} M\right)}{2} t_{0}^{2}\left(\sum_{i=1}^{n}\left(\sigma_{i}^{2}+a_{i}^{2}\right)+\left(X_{0}+\lambda\right) \sum_{i=1}^{n} \phi_{i}\right)} .
\end{aligned}
$$


Now we choose $t_{0}=\frac{\lambda}{\sum_{i=1}^{n}\left(\sigma_{i}^{2}+a_{i}^{2}\right)+\left(X_{0}+\lambda\right)\left(\sum_{i=1}^{n} \phi_{i}\right)+M \lambda / 3}$. Using the fact that $t_{0} M<3$, we have

$$
\begin{aligned}
\operatorname{Pr}\left(X_{n} \geq X_{0}+\lambda\right) & \leq e^{-t_{0} \lambda+t_{0}^{2}\left(\sum_{i=1}^{n}\left(\sigma_{i}^{2}+a_{i}^{2}\right)+\left(X_{0}+\lambda\right) \sum_{i=1}^{n} \phi_{i}\right) \frac{1}{2\left(1-t_{0} M / 3\right)}} \\
& =e^{-\frac{\lambda^{2}}{2\left(\sum_{i=1}^{n}\left(\sigma_{i}^{2}+a_{i}^{2}\right)+\left(X_{0}+\lambda\right)\left(\sum_{i=1}^{n} \phi_{i}\right)+M \lambda / 3\right)}}
\end{aligned}
$$

The proof of the theorem is complete.

Proof of Theorem 7.5. The proof is quite similar to that of Theorem 7.3. The following inequality still holds:

$$
\begin{aligned}
\mathrm{E}\left(e^{-t X_{i}} \mid \mathcal{F}_{i-1}\right) & =e^{-t \mathrm{E}\left(X_{i} \mid \mathcal{F}_{i-1}\right)+t a_{i}} \mathrm{E}\left(e^{-t\left(X_{i}-\mathrm{E}\left(X_{i} \mid \mathcal{F}_{i-1}\right)+a_{i}\right)} \mid \mathcal{F}_{i-1}\right) \\
& =e^{-t \mathrm{E}\left(X_{i} \mid \mathcal{F}_{i-1}\right)+t a_{i}} \sum_{k=0}^{\infty} \frac{t^{k}}{k !} \mathrm{E}\left(\left(\mathrm{E}\left(X_{i} \mid \mathcal{F}_{i-1}\right)-X_{i}-a_{i}\right)^{k} \mid \mathcal{F}_{i-1}\right) \\
& \leq e^{-t \mathrm{E}\left(X_{i} \mid \mathcal{F}_{i-1}\right)+\sum_{k=2}^{\infty} \frac{t^{k}}{k !} \mathrm{E}\left(\left(\mathrm{E}\left(X_{i} \mid \mathcal{F}_{i-1}\right)-X_{i}-a_{i}\right)^{k} \mid \mathcal{F}_{i-1}\right)} \\
& \leq e^{-t \mathrm{E}\left(X_{i} \mid \mathcal{F}_{i-1}\right)+\frac{g(t M)}{2} t^{2} \mathrm{E}\left(\left(X_{i}-\mathrm{E}\left(X_{i} \mid \mathcal{F}_{i-1}\right)-a_{i}\right)^{2}\right)} \\
& \leq e^{-t \mathrm{E}\left(X_{i} \mid \mathcal{F}_{i-1}\right)+\frac{g(t M)}{2} t^{2}\left(\operatorname{Var}\left(X_{i} \mid \mathcal{F}_{i-1}\right)+a_{i}^{2}\right)} \\
& \leq e^{-\left(t-\frac{g(t M)}{2} t^{2} \phi_{i}\right) X_{i-1}} e^{\frac{g(t M)}{2} t^{2}\left(\sigma_{i}^{2}+a_{i}^{2}\right)} .
\end{aligned}
$$

We now define $t_{i} \geq 0$, for $0 \leq i<n$, satisfying

$$
t_{i-1}=t_{i}-\frac{g\left(t_{n} M\right)}{2} \phi_{i} t_{i}^{2}
$$

$t_{n}$ will be defined later. Then, we have

$$
t_{0} \leq t_{1} \leq \cdots \leq t_{n}
$$

and

$$
\begin{aligned}
\mathrm{E}\left(e^{-t_{i} X_{i}} \mid \mathcal{F}_{i-1}\right) & \leq e^{-\left(t_{i}-\frac{g\left(t_{i} M\right)}{2} t_{i}^{2} \phi_{i}\right) X_{i-1}} e^{\frac{g\left(t_{i} M\right)}{2} t_{i}^{2}\left(\sigma_{i}^{2}+a_{i}^{2}\right)} \\
& \leq e^{-\left(t_{i}-\frac{g\left(t_{n} M\right)}{2} t_{i}^{2} \phi_{i}\right) X_{i-1}} e^{\frac{g\left(t_{n} M\right)}{2} t_{i}^{2}\left(\sigma_{i}^{2}+a_{i}^{2}\right)} \\
& =e^{-t_{i-1} X_{i-1}} e^{\frac{g\left(t_{n} M\right)}{2} t_{i}^{2}\left(\sigma_{i}^{2}+a_{i}^{2}\right)} .
\end{aligned}
$$


By Markov's inequality, we have

$$
\begin{aligned}
\operatorname{Pr}\left(X_{n} \leq X_{0}-\lambda\right) & =\operatorname{Pr}\left(-t_{n} X_{n} \geq-t_{n}\left(X_{0}-\lambda\right)\right) \\
& \leq e^{t_{n}\left(X_{0}-\lambda\right)} \mathrm{E}\left(e^{-t_{n} X_{n}}\right) \\
& =e^{t_{n}\left(X_{0}-\lambda\right)} \mathrm{E}\left(\mathrm{E}\left(e^{-t_{n} X_{n}} \mid \mathcal{F}_{n-1}\right)\right) \\
& \leq e^{t_{n}\left(X_{0}-\lambda\right)} \mathrm{E}\left(e^{-t_{n-1} X_{n-1}}\right) e^{\frac{g\left(t_{n} M\right)}{2} t_{n}^{2}\left(\sigma_{n}^{2}+a_{n}^{2}\right)} \\
& \vdots \\
& \leq e^{t_{n}\left(X_{0}-\lambda\right)} \mathrm{E}\left(e^{-t_{0} X_{0}}\right) e^{\sum_{i=1}^{n} \frac{g\left(t_{n} M\right)}{2} t_{i}^{2}\left(\sigma_{i}^{2}+a_{i}^{2}\right)} \\
& \leq e^{t_{n}\left(X_{0}-\lambda\right)-t_{0} X_{0}+\frac{t_{n}^{2}}{2} g\left(t_{n} M\right) \sum_{i=1}^{n}\left(\sigma_{i}^{2}+a_{i}^{2}\right)} .
\end{aligned}
$$

We note that

$$
\begin{aligned}
t_{0} & =t_{n}+\sum_{i=1}^{n}\left(t_{i-1}-t_{i}\right) \\
& =t_{n}-\sum_{i=1}^{n} \frac{g\left(t_{n} M\right)}{2} \phi_{i} t_{i}^{2} \\
& \geq t_{n}-\frac{g\left(t_{n} M\right)}{2} t_{n}^{2} \sum_{i=1}^{n} \phi_{i} .
\end{aligned}
$$

Thus, we have

$$
\begin{aligned}
\operatorname{Pr}\left(X_{n} \leq X_{0}-\lambda\right) & \leq e^{t_{n}\left(X_{0}-\lambda\right)-t_{0} X_{0}+\frac{t_{n}^{2}}{2} g\left(t_{n} M\right) \sum_{i=1}^{n}\left(\sigma_{i}^{2}+a_{i}^{2}\right)} \\
& \leq e^{t_{n}\left(X_{0}-\lambda\right)-\left(t_{n}-\frac{g\left(t_{n} M\right)}{2} t_{n}^{2}\right) X_{0}+\frac{t_{n}^{2}}{2} g\left(t_{n} M\right) \sum_{i=1}^{n}\left(\sigma_{i}^{2}+a_{i}^{2}\right)} \\
& =e^{-t_{n} \lambda+\frac{g\left(t_{n} M\right)}{2} t_{n}^{2}\left(\sum_{i=1}^{n}\left(\sigma_{i}^{2}+a_{i}^{2}\right)+\left(\sum_{i=1}^{n} \phi_{i}\right) X_{0}\right)} .
\end{aligned}
$$

We choose $t_{n}=\frac{\lambda}{\sum_{i=1}^{n}\left(\sigma_{i}^{2}+a_{i}^{2}\right)+\left(\sum_{i=1}^{n} \phi_{i}\right) X_{0}+M \lambda / 3}$. We have $t_{n} M<3$ and

$$
\begin{aligned}
\operatorname{Pr}\left(X_{n} \leq X_{0}-\lambda\right) & \leq e^{-t_{n} \lambda+t_{n}^{2}\left(\sum_{i=1}^{n}\left(\sigma_{i}^{2}+a_{i}^{2}\right)+\left(\sum_{i=1}^{n} \phi_{i}\right) X_{0}\right) \frac{1}{2\left(1-t_{n} M / 3\right)}} \\
& \leq e^{-\frac{\lambda^{2}}{2\left(\sum_{i=1}^{n}\left(\sigma_{i}^{2}+a_{i}^{2}\right)+X_{0}\left(\sum_{i=1}^{n} \phi_{i}\right)+M \lambda / 3\right)}} .
\end{aligned}
$$


It remains to verify that all $t_{i}$ are nonnegative. Indeed,

$$
\begin{aligned}
t_{i} & \geq t_{0} \\
& \geq t_{n}-\frac{g\left(t_{n} M\right)}{2} t_{n}^{2} \sum_{i=1}^{n} \phi_{i} \\
& \geq t_{n}\left(1-\frac{1}{2\left(1-t_{n} M / 3\right)} t_{n} \sum_{i=1}^{n} \phi_{i}\right) \\
& =t_{n}\left(1-\frac{\lambda}{2 X_{0}+\frac{\sum_{i=1}^{n}\left(\sigma_{i}^{2}+a_{i}^{2}\right)}{\sum_{i=1}^{n} \phi_{i}}}\right) \\
& \geq 0 .
\end{aligned}
$$

The proof of the theorem is complete.

\section{The Decision Tree and Relaxed Concentration Inequalities}

In this section, we will extend and generalize previous theorems to a martingale that is not strictly Lipschitz but is nearly Lipschitz. Namely, the (Lipschitz-like) assumptions are allowed to fail for relatively small subsets of the probability space, and we can still have similar but weaker concentration inequalities. Similar techniques have been introduced by $\mathrm{Kim}$ and $\mathrm{Vu}$ in their important work on deriving concentration inequalities for multivariate polynomials [Kim and $\mathrm{Vu} 00$ ]. The basic setup for decision trees can be found in [Alon and Spencer 92] and has been used in the work of Alon, Kim, and Spencer [Alon et al. 97]. Wormald [Wormald 99] considers martingales with a "stopping time" that has a similar flavor. Here we use a rather general setting, and we shall give a complete proof.

We are only interested in finite probability spaces, and we use the following computational model. The random variable $X$ can be evaluated by a sequence of decisions $Y_{1}, Y_{2}, \ldots, Y_{n}$. Each decision has finitely many outputs. The probability that an output is chosen depends on the previous history. We can describe the process by a decision tree $T$, a complete rooted tree with depth $n$. Each edge $u v$ of $T$ is associated with a probability $p_{u v}$ depending on the decision made from $u$ to $v$. Note that for any node $u$, we have

$$
\sum_{v} p_{u, v}=1
$$

We allow $p_{u v}$ to be zero and thus include the case of having fewer than $r$ outputs, for some fixed $r$. Let $\Omega_{i}$ denote the probability space obtained after the first $i$ decisions. Suppose that $\Omega=\Omega_{n}$ and $X$ is the random variable on $\Omega$. Let 
$\pi_{i}: \Omega \rightarrow \Omega_{i}$ be the projection mapping each point to the subset of points with the same first $i$ decisions. Let $\mathcal{F}_{i}$ be the $\sigma$-field generated by $Y_{1}, Y_{2}, \ldots, Y_{i}$. (In fact, $\mathcal{F}_{i}=\pi_{i}^{-1}\left(2^{\Omega_{i}}\right)$ is the full $\sigma$-field via the projection $\pi_{i}$.) The $\mathcal{F}_{i}$ form a natural filter:

$$
\{\emptyset, \Omega\}=\mathcal{F}_{0} \subset \mathcal{F}_{1} \subset \cdots \subset \mathcal{F}_{n}=\mathcal{F} .
$$

The leaves of the decision tree are exactly the elements of $\Omega$. Let $X_{0}, X_{1}, \ldots, X_{n}=$ $X$ denote the sequence of decisions to evaluate $X$. Note that $X_{i}$ is $\mathcal{F}_{i}$-measurable and can be interpreted as a labeling on nodes at depth $i$.

There is one-to-one correspondence between the following:

- A sequence of random variables $X_{0}, X_{1}, \ldots, X_{n}$ satisfying $X_{i}$ is $\mathcal{F}_{i}$-measurable, for $i=0,1, \ldots, n$.

- A vertex labeling of the decision tree $T, f: V(T) \rightarrow \mathbb{R}$.

In order to simplify and unify the proofs for various general types of martingales, here we introduce a definition for a function $f: V(T) \rightarrow \mathbb{R}$. We say $f$ satisfies an admissible condition $P$ if $P=\left\{P_{v}\right\}$ holds for every vertex $v$.

Here are examples of admissible conditions:

1. Supermartingale: For $1 \leq i \leq n$, we have

$$
\mathrm{E}\left(X_{i} \mid \mathcal{F}_{i-1}\right) \geq X_{i-1}
$$

Thus, the admissible condition $P_{u}$ holds if

$$
f(u) \leq \sum_{v \in C(u)} p_{u v} f(v)
$$

where $C(u)$ is the set of all children nodes of $u$ and $p_{u v}$ is the transition probability at the edge $u v$.

2. Submartingale: For $1 \leq i \leq n$, we have

$$
\mathrm{E}\left(X_{i} \mid \mathcal{F}_{i-1}\right) \leq X_{i-1}
$$

In this case, the admissible condition of the submartingale is

$$
f(u) \geq \sum_{v \in C(u)} p_{u v} f(v) .
$$

3. Martingale: For $1 \leq i \leq n$, we have

$$
\mathrm{E}\left(X_{i} \mid \mathcal{F}_{i-1}\right)=X_{i-1} .
$$


The admissible condition of the martingale is then

$$
f(u)=\sum_{v \in C(u)} p_{u v} f(v)
$$

4. c-Lipschitz: For $1 \leq i \leq n$, we have

$$
\left|X_{i}-X_{i-1}\right| \leq c_{i}
$$

The admissible condition of the c-Lipschitz property can be described as follows:

$$
|f(u)-f(v)| \leq c_{i}, \quad \text { for any child } v \in C(u),
$$

where the node $u$ is at level $i$ of the decision tree.

5. Bounded variance: For $1 \leq i \leq n$, we have

$$
\operatorname{Var}\left(X_{i} \mid \mathcal{F}_{i-1}\right) \leq \sigma_{i}^{2}
$$

for some constants $\sigma_{i}$.

The admissible condition of the bounded variance property can be described as

$$
\sum_{v \in C(u)} p_{u v} f^{2}(v)-\left(\sum_{v \in C(u)} p_{u v} f(v)\right)^{2} \leq \sigma_{i}^{2}
$$

6. General bounded variance: For $1 \leq i \leq n$, we have

$$
\operatorname{Var}\left(X_{i} \mid \mathcal{F}_{i-1}\right) \leq \sigma_{i}^{2}+\phi_{i} X_{i-1}
$$

where $\sigma_{i}$ and $\phi_{i}$ are nonnegative constants and $X_{i} \geq 0$. The admissible condition of the general bounded variance property can be described as follows:

$$
\sum_{v \in C(u)} p_{u v} f^{2}(v)-\left(\sum_{v \in C(u)} p_{u v} f(v)\right)^{2} \leq \sigma_{i}^{2}+\phi_{i} f(u), \quad \text { for } f(u) \geq 0,
$$

where $i$ is the depth of the node $u$.

7. Upper-bound: For $1 \leq i \leq n$, we have

$$
X_{i}-\mathrm{E}\left(X_{i} \mid \mathcal{F}_{i-1}\right) \leq a_{i}+M
$$


where $a_{i}$ and $M$ are nonnegative constants. The admissible condition of the upper bounded property can be described as follows:

$$
f(v)-\sum_{v \in C(u)} p_{u v} f(v) \leq a_{i}+M, \quad \text { for any child } v \in C(u),
$$

where $i$ is the depth of the node $u$.

8. Lower-bound: For $1 \leq i \leq n$, we have

$$
\mathrm{E}\left(X_{i} \mid \mathcal{F}_{i-1}\right)-X_{i} \leq a_{i}+M
$$

where $a_{i}$ and $M$ are nonnegative constants. The admissible condition of the lower bounded property can be described as follows:

$$
\sum_{v \in C(u)} p_{u v} f(v)-f(v) \leq a_{i}+M, \quad \text { for any child } v \in C(u),
$$

where $i$ is the depth of the node $u$.

For any labeling $f$ on $T$ and fixed vertex $r$, we can define a new labeling $f_{r}$ as follows:

$$
f_{r}(u)= \begin{cases}f(r) & \text { if } u \text { is a descedant of } r \\ f(u) & \text { otherwise. }\end{cases}
$$

A property $P$ is said to be invariant under subtree-unification if for a vertex $r$ and any tree labeling $f$ satisfying $P, f_{r}$ satisfies $P$.

We have the following theorem.

Theorem 8.I. The eight properties as stated in the preceding examples-supermartingale, submartingale, martingale, c-Lipschitz, bounded variance, general bounded variance, upper-bounded, and lower-bounded properties-are all invariant under subtree-unification.

Proof. We note that these properties are all admissible conditions. Let $P$ denote any one of these. For any node $u$, if $u$ is not a descendant of $r$, then $f_{r}$ and $f$ have the same value on $v$ and its children nodes. Hence, $P_{u}$ holds for $f_{r}$ since $P_{u}$ does for $f$.

If $u$ is a descendant of $r$, then $f_{r}(u)$ takes the same value as $f(r)$ as well as its children nodes. We verify $P_{u}$ in each case. Assume that $u$ is at level $i$ of the decision tree $T$. 
1. For supermartingale, submartingale, and martingale properties, we have

$$
\begin{aligned}
\sum_{v \in C(u)} p_{u v} f_{r}(v) & =\sum_{v \in C(u)} p_{u v} f(r) \\
& =f(r) \sum_{v \in C(u)} p_{u v} \\
& =f(r) \\
& =f_{r}(u) .
\end{aligned}
$$

Hence, $P_{u}$ holds for $f_{r}$.

2. For the c-Lipschitz property, we have

$$
\left|f_{r}(u)-f_{r}(v)\right|=0 \leq c_{i}, \quad \text { for any child } v \in C(u) .
$$

Again, $P_{u}$ holds for $f_{r}$.

3. For the bounded variance property, we have

$$
\begin{aligned}
& \sum_{v \in C(u)} p_{u v} f_{r}^{2}(v)-\left(\sum_{v \in C(u)} p_{u v} f_{r}(v)\right)^{2} \\
&=\sum_{v \in C(u)} p_{u v} f^{2}(r)-\left(\sum_{v \in C(u)} p_{u v} f(r)\right)^{2} \\
&=f^{2}(r)-f^{2}(r) \\
&=0 \\
& \leq \sigma_{i}^{2}
\end{aligned}
$$

4. For the second bounded variance property, we have

$$
\begin{aligned}
f_{r}(u) & =f(r) \geq 0 \\
\sum_{v \in C(u)} p_{u v} f_{r}^{2}(v)-\left(\sum_{v \in C(u)} p_{u v}\right. & \left.f_{r}(v)\right)^{2} \\
& =\sum_{v \in C(u)} p_{u v} f^{2}(r)-\left(\sum_{v \in C(u)} p_{u v} f(r)\right)^{2} \\
& =f^{2}(r)-f^{2}(r) \\
& =0 \\
& \leq \sigma_{i}^{2}+\phi_{i} f_{r}(u) .
\end{aligned}
$$


5. For the upper-bounded property, we have

$$
\begin{aligned}
f_{r}(v)-\sum_{v \in C(u)} p_{u v} f_{r}(v) & =f(r)-\sum_{v \in C(u)} p_{u v} f(r) \\
& =f(r)-f(r) \\
& =0 \\
& \leq a_{i}+M,
\end{aligned}
$$

for any child $v$ of $u$.

6. For the lower-bounded property, we have

$$
\begin{aligned}
\sum_{v \in C(u)} p_{u v} f_{r}(v)-f_{r}(v) & =\sum_{v \in C(u)} p_{u v} f(r)-f(r) \\
& =f(r)-f(r) \\
& =0 \\
& \leq a_{i}+M
\end{aligned}
$$

for any child $v$ of $u$.

Therefore, $P_{v}$ holds for $f_{r}$ and any vertex $v$.

For two admissible conditions $P$ and $Q$, we define $P Q$ to be the property that is only true when both $P$ and $Q$ are true. If both admissible conditions $P$ and $Q$ are invariant under subtree-unification, then $P Q$ is also invariant under subtree-unification.

For any vertex $u$ of the tree $T$, an ancestor of $u$ is a vertex lying on the unique path from the root to $u$. For an admissible condition $P$, the associated bad set $B_{i}$ over the $X_{i}$ is defined to be

$B_{i}=\left\{v \mid\right.$ the depth of $v$ is $i$, and $P_{u}$ does not hold for some ancestor $u$ of $\left.v\right\}$.

Lemma 8.2. For a filter $\mathbf{F}$,

$$
\{\emptyset, \Omega\}=\mathcal{F}_{0} \subset \mathcal{F}_{1} \subset \cdots \subset \mathcal{F}_{n}=\mathcal{F},
$$

suppose that each random variable $X_{i}$ is $\mathcal{F}_{i}$-measurable, for $0 \leq i \leq n$. For any admissible condition $P$, let $B_{i}$ be the associated bad set of $P$ over $X_{i}$. There are random variables $Y_{0}, \ldots, Y_{n}$ satisfying

(1) $Y_{i}$ is $\mathcal{F}_{i}$-measurable.

(2) $Y_{0}, \ldots, Y_{n}$ satisfy condition $P$.

(3) $\left\{x: Y_{i}(x) \neq X_{i}(x)\right\} \subset B_{i}$, for $0 \leq i \leq n$. 
Proof. We modify $f$ and define $f^{\prime}$ on $T$ as follows. For any vertex $u$,

$f^{\prime}(u)= \begin{cases}f(u) & \text { if } f \text { satisfies } P_{v} \text { for every ancestor } v \text { of } u \text { including } u \text { itself, } \\ f(v) & v \text { is the ancestor with smallest depth so that } f \text { fails } P_{v} .\end{cases}$

Let $S$ be the set of vertices $u$ satisfying

- $f$ fails $P_{u}$,

- $f$ satisfies $P_{v}$ for every ancestor $v$ of $u$.

It is clear that $f^{\prime}$ can be obtained from $f$ by a sequence of subtree-unifications, where $S$ is the set of the roots of subtrees. Furthermore, the order of subtreeunifications does not matter. Since $P$ is invariant under subtree-unifications, the number of vertices that $P$ fails decreases. Now we will show that $f^{\prime}$ satisfies $P$.

Suppose to the contrary that $f^{\prime}$ fails $P_{u}$ for some vertex $u$. Since $P$ is invariant under subtree-unifications, $f$ also fails $P_{u}$. By the definition, there is an ancestor $v$ (of $u$ ) in $S$. After the subtree-unification on the subtree rooted at $v, P_{u}$ is satisfied. This is a contradiction.

Let $Y_{0}, Y_{1}, \ldots, Y_{n}$ be the random variables corresponding to the labeling $f^{\prime}$. The $Y_{i}$ satisfy the desired properties in (1)-(3).

The following theorem generalizes Azuma's inequality. A similar but more restricted version can be found in [Kim and $\mathrm{Vu} 00]$.

Theorem 8.3. For a filter $\mathbf{F}$,

$$
\{\emptyset, \Omega\}=\mathcal{F}_{0} \subset \mathcal{F}_{1} \subset \cdots \subset \mathcal{F}_{n}=\mathcal{F}
$$

suppose that the random variable $X_{i}$ is $\mathcal{F}_{i}$-measurable, for $0 \leq i \leq n$. Let $B=B_{n}$ denote the bad set associated with the following admissible condition:

$$
\begin{aligned}
\mathrm{E}\left(X_{i} \mid \mathcal{F}_{i-1}\right) & =X_{i-1}, \\
\left|X_{i}-X_{i-1}\right| & \leq c_{i},
\end{aligned}
$$

for $1 \leq i \leq n$ where $c_{1}, c_{2}, \ldots, c_{n}$ are nonnegative numbers. Then, we have

$$
\operatorname{Pr}\left(\left|X_{n}-X_{0}\right| \geq \lambda\right) \leq 2 e^{-\frac{\lambda^{2}}{2 \sum_{i=1}^{n} c_{i}^{2}}}+\operatorname{Pr}(B)
$$

Proof. We use Lemma 8.2, which gives random variables $Y_{0}, Y_{1}, \ldots, Y_{n}$ satisfying properties (1)-(3) in the statement of Lemma 8.2. Then, it satisfies

$$
\begin{aligned}
\mathrm{E}\left(Y_{i} \mid \mathcal{F}_{i-1}\right) & =Y_{i-1}, \\
\left|Y_{i}-Y_{i-1}\right| & \leq c_{i} .
\end{aligned}
$$


In other words, $Y_{0}, \ldots, Y_{n}$ form a martingale that is $\left(c_{1}, \ldots, c_{n}\right)$-Lipschitz. By Azuma's inequality, we have

$$
\operatorname{Pr}\left(\left|Y_{n}-Y_{0}\right| \geq \lambda\right) \leq 2 e^{-\frac{\lambda^{2}}{2 \sum_{i=1}^{n} c_{i}^{2}}}
$$

Since $Y_{0}=X_{0}$ and $\left\{x: Y_{n}(x) \neq X_{n}(x)\right\} \subset B_{n}=B$, we have

$$
\begin{aligned}
\operatorname{Pr}\left(\left|X_{n}-X_{0}\right| \geq \lambda\right) & \leq \operatorname{Pr}\left(\left|Y_{n}-Y_{0}\right| \geq \lambda\right)+\operatorname{Pr}\left(X_{n} \neq Y_{n}\right) \\
& \leq 2 e^{-\frac{\lambda^{2}}{2 \sum_{i=1}^{n} c_{i}^{2}}}+\operatorname{Pr}(B) .
\end{aligned}
$$

For $\mathbf{c}=\left(c_{1}, c_{2}, \ldots, c_{n}\right)$, a vector with positive entries, a martingale is said to be near-c-Lipschitz with an exceptional probability $\eta$ if

$$
\sum_{i} \operatorname{Pr}\left(\left|X_{i}-X_{i-1}\right| \geq c_{i}\right) \leq \eta
$$

Theorem 8.3 can be restated as follows:

Theorem 8.4. For nonnegative values $c_{1}, c_{2}, \ldots, c_{n}$, suppose that a martingale $X$ is near-c-Lipschitz with an exceptional probability $\eta$. Then, $X$ satisfies

$$
\operatorname{Pr}(|X-\mathrm{E}(X)|<a) \leq 2 e^{-\frac{a^{2}}{2 \sum_{i=1}^{n} c_{i}^{2}}}+\eta .
$$

Now, we can apply the same technique to relax all the theorems in the previous sections.

Here are the relaxed versions of Theorems 6.3, 7.1, and 7.3.

Theorem 8.5. For a filter $\mathbf{F}$,

$$
\{\emptyset, \Omega\}=\mathcal{F}_{0} \subset \mathcal{F}_{1} \subset \cdots \subset \mathcal{F}_{n}=\mathcal{F},
$$

suppose that a random variable $X_{i}$ is $\mathcal{F}_{i}$-measurable, for $0 \leq i \leq n$. Let $B$ be the bad set associated with the following admissible conditions:

$$
\begin{aligned}
\mathrm{E}\left(X_{i} \mid \mathcal{F}_{i-1}\right) & \leq X_{i-1}, \\
\operatorname{Var}\left(X_{i} \mid \mathcal{F}_{i-1}\right) & \leq \sigma_{i}^{2}, \\
X_{i}-\mathrm{E}\left(X_{i} \mid \mathcal{F}_{i-1}\right) & \leq a_{i}+M,
\end{aligned}
$$

for some nonnegative constants $\sigma_{i}$ and $a_{i}$. Then, we have

$$
\operatorname{Pr}\left(X_{n} \geq X_{0}+\lambda\right) \leq e^{-\frac{\lambda^{2}}{2\left(\sum_{i=1}^{n}\left(\sigma_{i}^{2}+a_{i}^{2}\right)+M \lambda / 3\right)}}+\operatorname{Pr}(B) .
$$


Theorem 8.6. For a filter $\mathbf{F}$,

$$
\{\emptyset, \Omega\}=\mathcal{F}_{0} \subset \mathcal{F}_{1} \subset \cdots \subset \mathcal{F}_{n}=\mathcal{F}
$$

suppose that a nonnegative random variable $X_{i}$ is $\mathcal{F}_{i}$-measurable, for $0 \leq i \leq n$. Let $B$ be the bad set associated with the following admissible conditions:

$$
\begin{aligned}
\mathrm{E}\left(X_{i} \mid \mathcal{F}_{i-1}\right) & \leq X_{i-1}, \\
\operatorname{Var}\left(X_{i} \mid \mathcal{F}_{i-1}\right) & \leq \phi_{i} X_{i-1}, \\
X_{i}-\mathrm{E}\left(X_{i} \mid \mathcal{F}_{i-1}\right) & \leq M,
\end{aligned}
$$

for some nonnegative constants $\phi_{i}$ and $M$. Then, we have

$$
\operatorname{Pr}\left(X_{n} \geq X_{0}+\lambda\right) \leq e^{-\frac{\lambda^{2}}{2\left(\left(X_{0}+\lambda\right)\left(\sum_{i=1}^{n} \phi_{i}\right)+M \lambda / 3\right)}}+\operatorname{Pr}(B) .
$$

Theorem 8.7. For a filter $\mathbf{F}$,

$$
\{\emptyset, \Omega\}=\mathcal{F}_{0} \subset \mathcal{F}_{1} \subset \cdots \subset \mathcal{F}_{n}=\mathcal{F}
$$

suppose that a nonnegative random variable $X_{i}$ is $\mathcal{F}_{i}$-measurable, for $0 \leq i \leq n$. Let $B$ be the bad set associated with the following admissible conditions:

$$
\begin{aligned}
\mathrm{E}\left(X_{i} \mid \mathcal{F}_{i-1}\right) & \leq X_{i-1}, \\
\operatorname{Var}\left(X_{i} \mid \mathcal{F}_{i-1}\right) & \leq \sigma_{i}^{2}+\phi_{i} X_{i-1}, \\
X_{i}-\mathrm{E}\left(X_{i} \mid \mathcal{F}_{i-1}\right) & \leq a_{i}+M,
\end{aligned}
$$

for some nonnegative constants $\sigma_{i}, \phi_{i}, a_{i}$ and $M$. Then, we have

$$
\operatorname{Pr}\left(X_{n} \geq X_{0}+\lambda\right) \leq e^{-\frac{\lambda^{2}}{2\left(\sum_{i=1}^{n}\left(\sigma_{i}^{2}+a_{i}^{2}\right)+\left(X_{0}+\lambda\right)\left(\sum_{i=1}^{n} \phi_{i}\right)+M \lambda / 3\right)}}+\operatorname{Pr}(B) .
$$

For supermartingales, we have the following relaxed versions of Theorems 6.5, 7.2 , and 7.5 .

Theorem 8.8. For a filter $\mathbf{F}$,

$$
\{\emptyset, \Omega\}=\mathcal{F}_{0} \subset \mathcal{F}_{1} \subset \cdots \subset \mathcal{F}_{n}=\mathcal{F}
$$

suppose that a random variable $X_{i}$ is $\mathcal{F}_{i}$-measurable, for $0 \leq i \leq n$. Let $B$ be the bad set associated with the following admissible conditions:

$$
\begin{aligned}
\mathrm{E}\left(X_{i} \mid \mathcal{F}_{i-1}\right) & \geq X_{i-1}, \\
\operatorname{Var}\left(X_{i} \mid \mathcal{F}_{i-1}\right) & \leq \sigma_{i}^{2}, \\
\mathrm{E}\left(X_{i} \mid \mathcal{F}_{i-1}\right)-X_{i} & \leq a_{i}+M,
\end{aligned}
$$


for some nonnegative constants $\sigma_{i}, a_{i}$, and $M$. Then, we have

$$
\operatorname{Pr}\left(X_{n} \leq X_{0}-\lambda\right) \leq e^{-\frac{\lambda^{2}}{2\left(\sum_{i=1}^{n}\left(\sigma_{i}^{2}+a_{i}^{2}\right)+M \lambda / 3\right)}}+\operatorname{Pr}(B) .
$$

Theorem 8.9. For a filter $\mathbf{F}$,

$$
\{\emptyset, \Omega\}=\mathcal{F}_{0} \subset \mathcal{F}_{1} \subset \cdots \subset \mathcal{F}_{n}=\mathcal{F}
$$

suppose that a random variable $X_{i}$ is $\mathcal{F}_{i}$-measurable, for $0 \leq i \leq n$. Let $B$ be the bad set associated with the following admissible conditions:

$$
\begin{aligned}
\mathrm{E}\left(X_{i} \mid \mathcal{F}_{i-1}\right) & \geq X_{i-1}, \\
\operatorname{Var}\left(X_{i} \mid \mathcal{F}_{i-1}\right) & \leq \phi_{i} X_{i-1}, \\
\mathrm{E}\left(X_{i} \mid \mathcal{F}_{i-1}\right)-X_{i} & \leq M,
\end{aligned}
$$

for some nonnegative constants $\phi_{i}$ and $M$. Then, we have

$$
\operatorname{Pr}\left(X_{n} \leq X_{0}-\lambda\right) \leq e^{-\frac{\lambda^{2}}{2\left(X_{0}\left(\sum_{i=1}^{n} \phi_{i}\right)+M \lambda / 3\right)}}+\operatorname{Pr}(B),
$$

for all $\lambda \leq X_{0}$

Theorem 8.10. For a filter $\mathbf{F}$,

$$
\{\emptyset, \Omega\}=\mathcal{F}_{0} \subset \mathcal{F}_{1} \subset \cdots \subset \mathcal{F}_{n}=\mathcal{F}
$$

suppose that a nonnegative random variable $X_{i}$ is $\mathcal{F}_{i}$-measurable, for $0 \leq i \leq n$. Let $B$ be the bad set associated with the following admissible conditions:

$$
\begin{aligned}
\mathrm{E}\left(X_{i} \mid \mathcal{F}_{i-1}\right) & \geq X_{i-1}, \\
\operatorname{Var}\left(X_{i} \mid \mathcal{F}_{i-1}\right) & \leq \sigma_{i}^{2}+\phi_{i} X_{i-1}, \\
\mathrm{E}\left(X_{i} \mid \mathcal{F}_{i-1}\right)-X_{i} & \leq a_{i}+M,
\end{aligned}
$$

for some nonnegative constants $\sigma_{i}, \phi_{i}, a_{i}$, and $M$. Then, we have

$$
\operatorname{Pr}\left(X_{n} \leq X_{0}-\lambda\right) \leq e^{-\frac{\lambda^{2}}{2\left(\sum_{i=1}^{n}\left(\sigma_{i}^{2}+a_{i}^{2}\right)+X_{0}\left(\sum_{i=1}^{n} \phi_{i}\right)+M \lambda / 3\right)}}+\operatorname{Pr}(B),
$$

for $\lambda<X_{0}$. 


\section{A Generalized Polya's Urn Problem}

To see the powerful effect of the concentration and martingale inequalities in the previous sections, the best way is to check out some interesting applications. In this section we give the probabilistic analysis of the following process involving balls and bins:

For a fixed $0 \leq p<1$ and a positive integer $\kappa>1$, begin with $\kappa$ bins, each containing one ball and then introduce balls one at a time. For each new ball, with probability $p$, create a new bin and place the ball in that bin; otherwise, place the ball in an existing bin, such that the probability that the ball is placed in a bin is proportional to the number of balls in that bin.

Polya's urn problem (see [Johnson and Kotz 77]) is a special case of the above process with $p=0$ so new bins are never created. For the case of $p>0$, this infinite Polya process has a similar flavor as the preferential attachment scheme, one of the main models for generating the web graph among other information networks (see [Albert and Barabási 02, Barabási and Albert 99]).

In Section 9.1, we will show that the infinite Polya process generates a power law distribution so that the expected fraction of bins having $k$ balls is asymptotic to $c k^{-\beta}$, where $\beta=1+1 /(1-p)$ and $c$ is a constant. Then, the concentration result on the probabilistic error estimates for the power law distribution will be given in Section 9.2.

\section{I. The Expected Number of Bins with $k$ Balls}

To analyze the infinite Polya process, we let $n_{t}$ denote the number of bins at time $t$ and let $e_{t}$ denote the number of balls at time $t$. We have

$$
e_{t}=t+\kappa
$$

The number of bins $n_{t}$, however, is a sum of $t$ random indicator variables,

$$
n_{t}=\kappa+\sum_{i=1}^{t} s_{t}
$$

where

$$
\begin{aligned}
& \operatorname{Pr}\left(s_{j}=1\right)=p \\
& \operatorname{Pr}\left(s_{j}=0\right) \quad=1-p .
\end{aligned}
$$


It follows that

$$
\mathrm{E}\left(n_{t}\right)=\kappa+p t
$$

To get a handle on the actual value of $n_{t}$, we use the binomial concentration inequality as described in Theorem 3.2. Namely,

$$
\operatorname{Pr}\left(\left|n_{t}-\mathrm{E}\left(n_{t}\right)\right|>a\right) \leq e^{-a^{2} /(2 p t+2 a / 3)}
$$

Thus, $n_{t}$ is exponentially concentrated around $\mathrm{E}\left(n_{t}\right)$.

The problem of interest is the distribution of sizes of bins in the infinite Polya process.

Let $m_{k, t}$ denote the number of bins with $k$ balls at time $t$. First, we note that

$$
m_{1,0}=\kappa \text { and } m_{0, k}=0 .
$$

We wish to derive the recurrence for the expected value $\mathrm{E}\left(m_{k, t}\right)$. Note that a bin with $k$ balls at time $t$ could have come from two cases, either it was a bin with $k$ balls at time $t-1$ and no ball was added to it, or it was a bin with $k-1$ balls at time $t-1$ and a new ball was put in. Let $\mathcal{F}_{t}$ be the $\sigma$-algebra generated by all the possible outcomes at time $t$.

$$
\begin{aligned}
\mathrm{E}\left(m_{k, t} \mid \mathcal{F}_{t-1}\right) & =m_{k, t-1}\left(1-\frac{(1-p) k}{t+\kappa}\right)+m_{k-1, t-1}\left(\frac{(1-p)(k-1)}{t+\kappa-1}\right) \\
\mathrm{E}\left(m_{k, t}\right) & =\mathrm{E}\left(m_{k, t-1}\right)\left(1-\frac{(1-p) k}{t+\kappa-1}\right)+\mathrm{E}\left(m_{k-1, t-1}\right)\left(\frac{(1-p)(k-1)}{t+\kappa-1}\right) .
\end{aligned}
$$

For $t>0$ and $k=1$, we have

$$
\begin{gathered}
\mathrm{E}\left(m_{1, t} \mid \mathcal{F}_{t-1}\right)=m_{1, t-1}\left(1-\frac{(1-p)}{t+\kappa-1}\right)+p \\
\mathrm{E}\left(m_{1, t}\right)=\mathrm{E}\left(m_{1, t-1}\right)\left(1-\frac{(1-p)}{t+\kappa-1}\right)+p .
\end{gathered}
$$

To solve this recurrence, we use the following fact (see [Chung and Lu 04]):

Fact 9.I. For a sequence $\left\{a_{t}\right\}$ satisfying the recursive relation $a_{t+1}=\left(1-\frac{b_{t}}{t}\right) a_{t}+c_{t}$, $\lim _{t \rightarrow \infty} \frac{a_{t}}{t}$ exists and

$$
\lim _{t \rightarrow \infty} \frac{a_{t}}{t}=\frac{c}{1+b},
$$

provided that $\lim _{t \rightarrow \infty} b_{t}=b>0$ and $\lim _{t \rightarrow \infty} c_{t}=c$.

We proceed by induction on $k$ to show that $\lim _{t \rightarrow \infty} \mathrm{E}\left(m_{k, t}\right) / t$ has a limit $M_{k}$ for each $k$. 
The first case is $k=1$. In this case, we apply Fact 9.1 with $b_{t}=b=1-p$ and $c_{t}=c=p$ to deduce that $\lim _{t \rightarrow \infty} \mathrm{E}\left(m_{1, t}\right) / t$ exists and

$$
M_{1}=\lim _{t \rightarrow \infty} \frac{\mathrm{E}\left(m_{1, t}\right)}{t}=\frac{p}{2-p} .
$$

Now we assume that $\lim _{t \rightarrow \infty} \mathrm{E}\left(m_{k-1, t}\right) / t$ exists, and we apply the fact again with $b_{t}=b=k(1-p)$ and $c_{t}=\mathrm{E}\left(m_{k-1, t-1}\right)(1-p)(k-1) /(t+\kappa-1)$, so

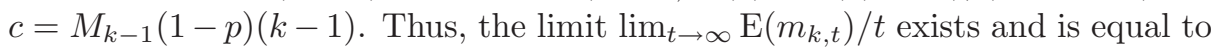

$$
M_{k}=M_{k-1} \frac{(1-p)(k-1)}{1+k(1-p)}=M_{k-1} \frac{k-1}{k+\frac{1}{1-p}} .
$$

Thus, we can write

$$
M_{k}=\frac{p}{2-p} \prod_{j=2}^{k} \frac{j-1}{j+\frac{1}{1-p}}=\frac{p}{2-p} \frac{\Gamma(k) \Gamma\left(2+\frac{1}{1-p}\right)}{\Gamma\left(k+1+\frac{1}{1-p}\right)},
$$

where $\Gamma(k)$ is the Gamma function.

We wish to show that the distribution of the bin sizes follows a power law with $M_{k} \propto k^{-\beta}$ (where $\propto$ means "is proportional to") for large $k$. If $M_{k} \propto k^{-\beta}$, then

$$
\frac{M_{k}}{M_{k-1}}=\frac{k^{-\beta}}{(k-1)^{-\beta}}=\left(1-\frac{1}{k}\right)^{\beta}=1-\frac{\beta}{k}+O\left(\frac{1}{k^{2}}\right) .
$$

From (9.3) we have

$$
\frac{M_{k}}{M_{k-1}}=\frac{k-1}{k+\frac{1}{1-p}}=1-\frac{1+\frac{1}{1-p}}{k+\frac{1}{1-p}}=1-\frac{1+\frac{1}{1-p}}{k}+O\left(\frac{1}{k^{2}}\right) .
$$

Thus, we have an approximate power-law with

$$
\beta=1+\frac{1}{1-p}=2+\frac{p}{1-p} .
$$

\subsection{Concentration on the Number of Bins with $k$ Balls}

Since the expected value can be quite different from the actual number of bins with $k$ balls at time $t$, we give a (probabilistic) estimate of the difference.

We will prove the following theorem.

Theorem 9.2. For the infinite Polya process, asymptotically almost surely the number of bins with $k$ balls at time $t$ is

$$
M_{k}(t+\kappa)+O\left(2 \sqrt{k^{3}(t+\kappa) \ln (t+\kappa)}\right) .
$$


Recall that $M_{1}=\frac{p}{2-p}$ and

$$
M_{k}=\frac{p}{2-p} \frac{\Gamma(k) \Gamma\left(2+\frac{1}{1-p}\right)}{\Gamma\left(k+1+\frac{1}{1-p}\right)}=O\left(k^{-\left(1+\frac{1}{1-p}\right)}\right),
$$

for $k \geq 2$. In other words, almost surely the distribution of the bin sizes for the infinite Polya process follows a power law with the exponent $\beta=1+\frac{1}{1-p}$.

Proof. We have shown that

$$
\lim _{t \rightarrow \infty} \frac{\mathrm{E}\left(m_{k, t}\right)}{t}=M_{k}
$$

where $M_{k}$ is defined recursively in (9.3). It is sufficient to show that $m_{k, t}$ concentrates on the expected value.

We shall prove the following claim.

Claim 9.3. For any fixed $k \geq 1$ and for any $c>0$ with probability at least $1-2(t+$ $\kappa+1)^{k-1} e^{-c^{2}}$, we have

$$
\left|m_{k, t}-M_{k}(t+\kappa)\right| \leq 2 k c \sqrt{t+\kappa} .
$$

To see that the claim implies Theorem 9.2, we choose $c=\sqrt{k \ln (t+\kappa)}$. Note that

$$
2(t+\kappa)^{k-1} e^{-c^{2}}=2(t+\kappa+1)^{k-1}(t+\kappa)^{-k}=o(1) .
$$

From Claim 9.3, with probability $1-o(1)$, we have

$$
\left|m_{k, t}-M_{k}(t+\kappa)\right| \leq 2 \sqrt{k^{3}(t+\kappa) \ln (t+\kappa)},
$$

as desired.

It remains to prove the claim.

Proof of Claim 9.3. We shall prove it by induction on $k$.

The base case of $k=1$. For $k=1$, from equation (9.2), we have

$$
\begin{aligned}
\mathrm{E}\left(m_{1, t}\right. & \left.-M_{1}(t+\kappa) \mid \mathcal{F}_{t-1}\right) \\
& =\mathrm{E}\left(m_{1, t} \mid \mathcal{F}_{t-1}\right)-M_{1}(t+\kappa) \\
& =m_{1, t-1}\left(1-\frac{1-p}{t+\kappa-1}\right)+p-M_{1}(t+\kappa-1)-M_{1} \\
& =\left(m_{1, t-1}-M_{1}(t+\kappa-1)\right)\left(1-\frac{1-p}{t+\kappa-1}\right)+p-M_{1}(1-p)-M_{1} \\
& =\left(m_{1, t-1}-M_{1}(t+\kappa-1)\right)\left(1-\frac{1-p}{t+\kappa-1}\right)
\end{aligned}
$$

since $p-M_{1}(1-p)-M_{1}=0$. 
Let $X_{1, t}=\frac{m_{1, t}-M_{1}(t+\kappa)}{\prod_{j=1}^{t}\left(1-\frac{1-p}{j+\kappa-1}\right)}$. We consider the martingale formed by $1=X_{1,0}, X_{1,1}, \ldots, X_{1, t}$. We have

$$
\begin{aligned}
X_{1, t}- & X_{1, t-1} \\
= & \frac{m_{1, t}-M_{1}(t+\kappa)}{\prod_{j=1}^{t}\left(1-\frac{1-p}{j+\kappa-1}\right)}-\frac{m_{1, t-1}-M_{1}(t+\kappa-1)}{\prod_{j=1}^{t-1}\left(1-\frac{1-p}{j+\kappa-1}\right)} \\
= & \frac{1}{\prod_{j=1}^{t}\left(1-\frac{1-p}{j+\kappa-1}\right)} \\
& \times\left[\left(m_{1, t}-M_{1}(t+\kappa)\right)-\left(m_{1, t-1}-M_{1}(t+\kappa-1)\right)\left(1-\frac{1-p}{t+\kappa-1}\right)\right] \\
= & \frac{1}{\prod_{j=1}^{t}\left(1-\frac{1-p}{j+\kappa-1}\right)} \\
& \times\left[\left(m_{1, t}-m_{1, t-1}\right)+\frac{1-p}{t+\kappa-1}\left(m_{1, t-1}-M_{1}(t+\kappa-1)\right)-M_{1}\right] .
\end{aligned}
$$

We note that $\left|m_{1, t}-m_{1, t-1}\right| \leq 1, m_{1, t-1} \leq t$ and $M_{1}=\frac{p}{2-p}<1$. We have

$$
\left|X_{1, t}-X_{1, t-1}\right| \leq \frac{1}{\prod_{j=1}^{t}\left(1-\frac{1-p}{j+\kappa-1}\right)} .
$$

Since $\left|m_{1, t}-m_{1, t-1}\right| \leq 1$, we have

$$
\begin{aligned}
\operatorname{Var}\left(m_{1, t} \mid \mathcal{F}_{t-1}\right) & \leq \mathrm{E}\left(\left(m_{1, t}-m_{1, t-1}\right)^{2} \mid \mathcal{F}_{t-1}\right) \\
& \leq 1 .
\end{aligned}
$$

Therefore, we have the following upper bound for $\operatorname{Var}\left(X_{1, t} \mid \mathcal{F}_{t-1}\right)$ :

$$
\begin{aligned}
\operatorname{Var}\left(X_{1, t} \mid \mathcal{F}_{t-1}\right) & =\operatorname{Var}\left(\left(m_{1, t}-M_{1}(t+\kappa)\right) \frac{1}{\prod_{j=1}^{t}\left(1-\frac{1-p}{j+\kappa-1}\right)} \mid \mathcal{F}_{t-1}\right) \\
& =\frac{1}{\prod_{j=1}^{t}\left(1-\frac{1-p}{j+\kappa-1}\right)^{2}} \operatorname{Var}\left(m_{1, t}-M_{1}(t+\kappa) \mid \mathcal{F}_{t-1}\right) \\
& =\frac{1}{\prod_{j=1}^{t}\left(1-\frac{1-p}{j+\kappa-1}\right)^{2}} \operatorname{Var}\left(m_{1, t} \mid \mathcal{F}_{t-1}\right) \\
& \leq \frac{1}{\prod_{j=1}^{t}\left(1-\frac{1-p}{j+\kappa-1}\right)^{2}} .
\end{aligned}
$$

We apply Theorem 6.2 on the martingale $\left\{X_{1, t}\right\}$ with $\sigma_{i}^{2}=\frac{4}{\prod_{j=1}^{i}\left(1-\frac{1-p}{j+\kappa-1}\right)^{2}}$, $M=\frac{4}{\prod_{j=1}^{t}\left(1-\frac{1-p}{j+\kappa-1}\right)}$, and $a_{i}=0$. We have

$$
\operatorname{Pr}\left(X_{1, t} \geq \mathrm{E}\left(X_{1, t}\right)+\lambda\right) \leq e^{-\frac{\lambda^{2}}{2\left(\sum_{i=1}^{t} \sigma_{i}^{2}+M \lambda / 3\right)}} .
$$


Here, $\mathrm{E}\left(X_{1, t}\right)=X_{1,0}=1$. We will use the following approximation:

$$
\begin{aligned}
\prod_{j=1}^{i}\left(1-\frac{1-p}{j+\kappa-1}\right) & =\prod_{j=1}^{i} \frac{j+\kappa-2+p}{j+\kappa-1} \\
& =\frac{\Gamma(\kappa) \Gamma(i+\kappa-1+p)}{\Gamma(\kappa-1+p) \Gamma(i+\kappa)} \\
& \approx C(i+\kappa)^{-1+p}
\end{aligned}
$$

where $C=\frac{\Gamma(\kappa)}{\Gamma(\kappa-1+p)}$ is a constant depending only on $p$ and $\kappa$.

For any $c>0$, we choose $\lambda=\frac{4 c \sqrt{t+\kappa}}{\prod_{j=1}^{t}\left(1-\frac{1-p}{j}\right)} \approx 4 C^{-1} c t^{3 / 2-p}$. We have

$$
\begin{aligned}
\sum_{i=1}^{t} \sigma_{i}^{2} & =\sum_{i=1}^{t} \frac{4}{\prod_{j=1}^{i}\left(1-\frac{1-p}{j}\right)^{2}} \\
& \approx \sum_{i=1}^{t} 4 C^{-2}(i+\kappa)^{2-2 p} \\
& \approx \frac{4 C^{-2}}{3-2 p}(t+\kappa)^{3-2 p} \\
& <4 C^{-2}(t+\kappa)^{3-2 p}
\end{aligned}
$$

We note that

$$
M \lambda / 3 \approx \frac{8}{3} C^{-2} c t^{5 / 2-2 p}<2 C^{-2} t^{3-2 p},
$$

provided that $4 c / 3<\sqrt{t+\kappa}$. We have

$$
\begin{aligned}
\operatorname{Pr}\left(X_{1, t} \geq 1+\lambda\right) & \leq e^{-\frac{\lambda^{2}}{2\left(\sum_{i=1}^{t} \sigma_{i}^{2}+M \lambda / 3\right)}} \\
& <e^{-\frac{16 C^{-2} c^{2} t^{3-2 p}}{8 C^{-2} t^{3-2 p}+2 C^{-2}(t+\kappa)^{3-2 p}}} \\
& <e^{-c^{2}}
\end{aligned}
$$

Since 1 is much smaller than $\lambda$, we can replace $1+\lambda$ by $\lambda$ without loss of generality. Thus, with probability at least $1-e^{-c^{2}}$, we have

$$
X_{1, t} \leq \lambda
$$

Similarly, with probability at least $1-e^{-c^{2}}$, we have

$$
m_{1, t}-M_{1}(t+\kappa) \leq 2 c \sqrt{t+\kappa} .
$$

We remark that inequality (9.6) holds for any $c>0$. In fact, it is trivial when $4 c / 3>\sqrt{t+\kappa}$, since $\left|m_{1, t}-M_{1}(t+\kappa)\right| \leq 2 t$ always holds. 
Similarly, by applying Theorem 6.6 on the martingale, the lower bound

$$
m_{1, t}-M_{1}(t+\kappa) \geq-2 c \sqrt{t+\kappa}
$$

holds with probability at least $1-e^{-c^{2}}$.

We have proved the claim for $k=1$.

The inductive step. Suppose that the claim holds for $k-1$. For $k$, we define

$$
X_{k, t}=\frac{m_{k, t}-M_{k}(t+\kappa)-2(k-1) c \sqrt{t+\kappa}}{\prod_{j=1}^{t}\left(1-\frac{(1-p) k}{j+\kappa-1}\right)} .
$$

We have

$$
\begin{aligned}
\mathrm{E}\left(m_{k, t}-M_{k}(t+\kappa)-2(k-1) c \sqrt{t+\kappa} \mid \mathcal{F}_{t-1}\right) & \\
= & \mathrm{E}\left(m_{k, t} \mid \mathcal{F}_{t-1}\right)-M_{k}(t+\kappa)-2(k-1) c \sqrt{t+\kappa} \\
= & m_{k, t-1}\left(1-\frac{(1-p) k}{t+\kappa-1}\right)+m_{k-1, t-1}\left(\frac{(1-p)(k-1)}{t+\kappa-1}\right) \\
& \quad-M_{k}(t+\kappa)-2(k-1) c \sqrt{t+\kappa} .
\end{aligned}
$$

By the induction hypothesis, with probability at least $1-2 t^{k-2} e^{-c^{2}}$, we have

$$
\left|m_{k-1, t-1}-M_{k-1}(t+\kappa)\right| \leq 2(k-1) c \sqrt{t+\kappa} .
$$

By using this estimate, with probability at least $1-2 t^{k-2} e^{-c^{2}}$, we have

$$
\begin{aligned}
\mathrm{E}\left(m_{k, t}\right. & \left.-M_{k}(t+\kappa)-2(k-1) c \sqrt{t+\kappa} \mid \mathcal{F}_{t-1}\right) \\
& \leq\left(1-\frac{(1-p) k}{t}\right)\left(m_{k, t-1}-M_{k}(t+\kappa-1)-2(k-1) c \sqrt{t+\kappa-1}\right),
\end{aligned}
$$

by using the fact that $M_{k} \leq M_{k-1}$ as seen in (9.3).

Therefore, $0=X_{k, 0}, X_{k, 1}, \cdots, X_{k, t}$ forms a submartingale with failure probability at most $2 t^{k-2} e^{-c^{2}}$.

Similar to inequalities (9.4) and (9.5), it can be easily shown that

$$
\left|X_{k, t}-X_{k, t-1}\right| \leq \frac{4}{\prod_{j=1}^{t}\left(1-\frac{(1-p) k}{j+\kappa-1}\right)}
$$

and

$$
\operatorname{Var}\left(X_{k, t} \mid \mathcal{F}_{t-1}\right) \leq \frac{4}{\prod_{j=1}^{t}\left(1-\frac{(1-p) k}{j+\kappa-1}\right)^{2}}
$$


We apply Theorem 8.6 on the submartingale with $\sigma_{i}^{2}=\frac{4}{\prod_{j=1}^{i}\left(1-\frac{(1-p) k}{j+\kappa-1}\right)^{2}}, M=$ $\frac{4}{\prod_{j=1}^{t}\left(1-\frac{(1-p) \kappa}{j+\kappa-1}\right)}$, and $a_{i}=0$. We have

$$
\operatorname{Pr}\left(X_{k, t} \geq \mathrm{E}\left(X_{k, t}\right)+\lambda\right) \leq e^{-\frac{\lambda^{2}}{2\left(\sum_{i=1}^{t} \sigma_{i}^{2}+M \lambda / 3\right)}}+\operatorname{Pr}(B),
$$

where $\operatorname{Pr}(B) \leq t^{k-1} e^{-c^{2}}$ by the induction hypothesis.

Here, $\mathrm{E}\left(X_{k, t}\right)=X_{k, 0}=0$. We will use the following approximation:

$$
\begin{aligned}
\prod_{j=1}^{i}\left(1-\frac{(1-p) k}{j+\kappa-1}\right) & =\prod_{j=1}^{i} \frac{j-(1-p) k}{j+\kappa-1} \\
& =\frac{\Gamma(\kappa)}{\Gamma(1-(1-p) k)} \frac{\Gamma(i+1-(1-p) k)}{\Gamma(i+\kappa)} \\
& \approx C_{k}(i+\kappa)^{-(1-p) k}
\end{aligned}
$$

where $C_{k}=\frac{\Gamma(\kappa)}{\Gamma(1-(1-p) k)}$ is a constant depending only on $k, p$, and $\kappa$.

For any $c>0$, we choose $\lambda=\frac{4 c \sqrt{t+\kappa}}{\prod_{j=1}^{t}\left(1-\frac{(1-p) k}{j}\right)} \approx 4 C_{k}^{-1} c t^{3 / 2-p}$. We have

$$
\begin{aligned}
\sum_{i=1}^{t} \sigma_{i}^{2} & =\sum_{i=1}^{t} \frac{4}{\prod_{j=1}^{i}\left(1-\frac{(1-p) k}{j}\right)^{2}} \\
& \approx \sum_{i=1}^{t} 4 C_{k}^{-2}(i+\kappa)^{2 k(1-p)} \\
& \approx \frac{4 C_{k}^{-2}}{1+2 k(1-p)}(t+\kappa)^{1+2 k(1-p)} \\
& <4 C_{k}^{-2}(t+\kappa)^{1+2 k(1-p)} .
\end{aligned}
$$

We note that

$$
M \lambda / 3 \approx \frac{8}{3} C_{k}^{-2} c(t+\kappa)^{1 / 2+2(1-p)}<2 C_{k}^{-2}(t+\kappa)^{1+2(1-p)},
$$

provided that $4 c / 3<\sqrt{t+\kappa}$. We have

$$
\begin{aligned}
\operatorname{Pr}\left(X_{k, t} \geq \lambda\right) & \leq e^{-\frac{\lambda^{2}}{2\left(\sum_{i=1}^{t} \sigma_{i}^{2}+M \lambda / 3\right)}}+\operatorname{Pr}(B) \\
& <e^{-\frac{16 C_{k}^{-2} c^{2}(t+\kappa)^{1+2 k(1-p)}}{8 C_{k}^{-2}(t+\kappa)^{1+2(1-p)}+2 C_{k}^{-2}(t+\kappa)^{1+2(1-p)}}}+\operatorname{Pr}(B) \\
& <e^{-c^{2}}+(t+\kappa)^{k-1} e^{-c^{2}} \\
& \leq(t+\kappa+1)^{k-1} e^{-c^{2}} .
\end{aligned}
$$


With probability at least $1-(t+\kappa+1)^{k-1} e^{-c^{2}}$, we have

$$
X_{k, t} \leq \lambda
$$

Equivalently, with probability at least $1-(t+\kappa+1)^{k-1} e^{-c^{2}}$, we have

$$
m_{k, t}-M_{k}(t+\kappa) \leq 2 c k \sqrt{t+\kappa} .
$$

We remark that inequality (9.9) holds for any $c>0$. In fact, it is trivial when $4 c / 3>\sqrt{t+\kappa}$, since $\left|m_{k, t}-M_{k}(t+\kappa)\right| \leq 2(t+\kappa)$ always holds.

To obtain the lower bound, we consider

$$
X_{k, t}^{\prime}=\frac{m_{k, t}-M_{k}(t+\kappa)+2(k-1) c \sqrt{t+\kappa}}{\prod_{j=1}^{t}\left(1-\frac{(1-p) k}{j+\kappa-1}\right)} .
$$

It can be shown easily that $X_{k, t}^{\prime}$ is nearly a supermartingale. Similarly, by applying Theorem 8.9 to $X_{k, t}^{\prime}$, the lower bound

$$
m_{k, t}-M_{k}(t+\kappa) \geq-2 k c \sqrt{t+\kappa}
$$

holds with probability at least $1-(t+\kappa+1)^{k-1} e^{-c^{2}}$.

Together these prove the statement for $k$. The proof of Theorem 9.2 is complete.

The above methods for proving concentration of the power law distribution for the infinite Polya process can easily be carried out for many other problems. One of the most popular models for generating random graphs (which simulate web graphs and various information networks) is the so-called preferential attachment scheme. The problem on the degree distribution of the preferential attachment scheme can be viewed as a variation of the Polya process as we will see. Before we proceed, we first give a short description for the preferential attachment scheme [Aiello et al. 02, Mitzenmacher 04]:

- With probability $p$, for some fixed $p$, add a new vertex $v$, and add an edge $\{u, v\}$ from $v$ by randomly and independently choosing $u$ in proportion to the degree of $u$ in the current graph. The initial graph, say, is one single vertex with a loop.

- Otherwise, add a new edge $\{r, s\}$ by independently choosing vertices $r$ and $s$ with probability proportional to their degrees. Here, $r$ and $s$ could be the same vertex.

The above preferential attachment scheme can be rewritten as the following variation of the Polya process: 
- Start with one bin containing one ball.

- At each step, with probability $p$, add two balls, one to a new bin and one to an existing bin with probability proportional to the bin size. With probability $1-p$, add two balls, each of which is independently placed in an existing bin with probability proportional to the bin size.

As we can see, the bins are the vertices; at each time step the bins that the two balls are placed are associated with an edge; the bin size is exactly the degree of the vertex.

It is not difficult to show the expected degrees of the preferential attachment model satisfy a power law distribution with exponent $1+2 /(2-p)$ (see [Aiello et al. 02, Mitzenmacher 04]). The concentration results for the power law degree distribution of the preferential attachment scheme can be proved in a very similar way as what we have done in this section for the Polya process. The details of the proof can be found in a forthcoming book [Chung and Lu 06].

Acknowledgements. The research for this paper was supported in part by NSF grants DMS 0100472 and ITR 0205061.

\section{References}

[Abello et al. 98] J. Abello, A. Buchsbaum, and J. Westbrook. "A Functional Approach to External Graph Algorithms." In Algorithm-ESA '98: 6th Annual European Symposium, Venice, Italy, August 24-26, 1998, Proceedings, pp. 332-343, Lecture Notes in Computer Science 1461. Berlin: Springer, 1998.

[Aiello et al. 00] W. Aiello, F. Chung, and L. Lu. "A Random Graph Model for Massive Graphs." In Proceedings of the Thirty-Second Annual ACM Symposium on Theory of Computing, pp. 171-180. New York: ACM Press, 2000.

[Aiello et al. 02] W. Aiello, F. Chung, and L. Lu. "Random Evolution in Massive Graphs." Extended abstract appeared in The 42th Annual Symposium on Foundation of Computer Sciences, October, 2001. Paper version appeared in Handbook of Massive Data Sets, edited by J. Abello, P. M. Pardalos, and M. G. Reinde, pp. 97-122. Dordrecht: Kluwer Academic Publishers, 2002.

[Albert and Barabási 02] R. Albert and A.-L. Barabási. "Statistical Mechanics of Complex Networks." Review of Modern Physics 74 (2002), 47-97.

[Alon and Spencer 92] N. Alon and J. H. Spencer. The Probabilistic Method. New York: Wiley and Sons, 1992.

[Barabási and Albert 99] A.-L. Barabási and R. Albert. "Emergence of Scaling in Random Networks." Science 286 (1999), 509-512.

[Alon et al. 97] N. Alon, J.-H. Kim, and J. H. Spencer. "Nearly Perfect Matchings in Regular Simple Hypergraphs." Israel J. Math. 100 (1997), 171-187. 
[Chernoff 81] H. Chernoff. "A Note on an Inequality Involving the Normal Distribution." Ann. Probab. 9 (1981), 533-535.

[Chung and Lu 02a] F. Chung and L. Lu. "The Average Distances in Random Graphs with Given Expected Degrees." Proceeding of National Academy of Science 99 (2002), 15879-15882.

[Chung and Lu 02b] F. Chung and L. Lu. "Connected Components in Random Graphs with Given Expected Degree Sequences." Annals of Combinatorics 6 (2002), 125145.

[Chung and Lu 04] F. Chung and L. Lu. "Coupling Online and Offline Analyses for Random Power Law Graphs." Internet Mathematics 1:4 (2004), 409-461.

[Chung and Lu 06] F. Chung and L. Lu. Complex Graphs and Networks. Manuscript, 2006.

[Chung et al. 03a] F. Chung, S. Handjani and D. Jungreis. "Generalizations of Polya's Urn Problem." Annals of Combinatorics 7 (2003), 141-153.

[Chung et al. 03b] F. Chung, L. Lu and V. Vu. "The Spectra of Random Graphs with Given Expected Degrees." Proceedings of National Academy of Sciences 100:11 (2003), 6313-6318.

[Feller 71] W. Feller. "Martingales." In An Introduction to Probability Theory and its Applications, Vol. 2. New York: Wiley, 1971.

[Graham et al. 94] R. L. Graham, D. E. Knuth, and O. Patashnik. Concrete Mathematics, Second edition. Reading, MA: Addison-Wesley Publishing Company, 1994.

[Janson et al. 00] S. Janson, T. Łuczak, and A. Rucínski. Random Graphs. New York: Wiley-Interscience, 2000.

[Johnson and Kotz 77] N. Johnson and S. Kotz. Urn Models and Their Applications: An Approach to Modern Discrete Probability Theory. New York: Wiley, 1977.

[Kim and Vu 00] J. H. Kim and V. Vu. "Concentration of Multivariate Polynomials and its Applications." Combinatorica 20:3 (2000), 417-434.

[McDiarmid 98] C. McDiarmid. "Concentration." In Probabilistic Methods for Algorithmic Discrete Mathematics, edited by M. Habib, C. McDiarmid, J. RamirezAlfonsin, and B. Reed, pp. 195-248, Algorithms and Combinatorics 16. Berlin: Springer, 1998.

[Mitzenmacher 04] M. Mitzenmacher. "A Brief History of Generative Models for Power Law and Lognormal Distribution." Internet Mathematics 1:2 (2004), 226-251.

[Wormald 99] N. C. Wormald. "The Differential Equation Method for Random Processes and Greedy Algorithms." In Lectures on Approximation and Randomized Algorithms, edited by M. Karonski and H. J. Proemel, pp. 73-155. Warsaw: PWN, 1999. 
Fan Chung, Department of Mathematics, University of California, San Diego, 9500 Gilman Drive, 0012, La Jolla, CA 92093-0112 (fan@ucsd.edu)

Linyuan Lu, Department of Mathematics, University of South Carolina, Columbia, SC 29208 (lu@math.sc.edu)

Received August 24, 2005; accepted December 10, 2005. 